DOI: $10.24850 /$ j-tyca-2021-04-02

Artículos

\title{
Una revisión de las metodologías para estimar el coeficiente de pérdidas en curvas de tuberías bajo flujo turbulento
}

\section{A review of the methodologies for estimating the coefficient of losses in pipe curves under turbulent flow}

José Juan Villegas-León1, ORCID: https://orcid.org/0000-0001-65931373

Alvaro Alberto López-Lambraño², ORCID: https://orcid.org/0000-00027899-7985

Carlos Fuentes3, ORCID: https://orcid.org/0000-0003-4335-6413

Darío Aranda-Pérez ${ }^{4}$, ORCID: https://orcid.org/0000-0002-8883-9921

Alvaro Alberto López-Ramos5, ORCID: https://orcid.org/0000-00031116-4089

${ }^{1}$ Facultad de Ingeniería, Arquitectura y Diseño, Universidad Autónoma de Baja California, Ensenada, Baja California, México, jvillegas82@uabc.edu.mx

2Facultad de Ingeniería, Arquitectura y Diseño, Universidad Autónoma de Baja California, Ensenada, Baja California, México, alopezl@uabc.edu.mx/ Hidrus, S.A. de C.V., Ensenada, Baja California, México, 
alopezl@hidrusmx.com/Grupo Hidrus S.A.S., Montería, Colombia, altoti@gmail.com

3Instituto Mexicano de Tecnología del Agua, Jiutepec, Morelos, México, cbfuentesr@gmail.com

${ }^{4}$ Facultad de Ingeniería, Arquitectura y Diseño, Universidad Autónoma de Baja California, Ensenada, Baja California, México, dario.aranda@uabc.edu.mx

${ }^{5}$ Grupo Hidrus S.A.S., Montería, Colombia/ Hidrus, S.A. de C.V., Ensenada, Baja California, México/ Escuela de Ingeniería y Arquitectura, Facultad de Ingeniería Civil, Universidad Pontificia Bolivariana, Montería, Córdoba, Colombia, alvaro.lopezr@upb.edu.co

Autor para correspondencia: Alvaro Alberto López-Lambraño, altoti@gmail.com, alopezl@hidrusmx.com

\section{Resumen}

Se presenta una revisión de las metodologías reportadas en la literatura para estimar el coeficiente de pérdidas en curvas simples de tuberías e instaladas en serie con forma en $L, S, U$ y $Z$. La información se expone organizada en orden cronológico y homogenizada para su comparación, proporcionando el coeficiente en función del ángulo de la curva, de la relación del radio con respecto al diámetro interno y del número de Reynolds. A partir de este análisis, se exhibe un sumario, donde se contrastan características y parámetros considerados en cada 
metodología, así como la presentación de resultados y especificaciones de validez. Con ello, se ha encontrado que se dispone de metodologías diferentes para obtener el coeficiente de pérdidas, mediante las cuales se obtienen valores distintos, que pueden deberse principalmente a desigualdades en el material analizado y en los factores adoptados en las investigaciones. Las curvas simples e instaladas en serie más estudiadas son de ángulo de $90^{\circ}$ con condición gradual y en forma-U (180 $)$, respectivamente. Los trabajos más sugeridos para estimar el coeficiente en curvas son los de Kirchbach (1929) e Ito (1959, 1960); sin embargo, en el manual de Idel'chik (1966) se presenta la más amplia información para todos los dispositivos estudiados.

Palabras clave: curvas de tuberías, pérdida de energía, coeficiente de pérdida, flujo turbulento, flujo en tuberías, modelación hidráulica.

\section{Abstract}

A review of the methodologies reported in the literature is presented to estimate the coefficient of losses in simple pipe curves and installed in series with L-shape, S, $U$ and $Z$. The information is presented organized in chronological order and homogenized for comparison, providing the coefficient according to the angle of the curve, the ratio of the radius to the internal diameter and the Reynolds number. From this analysis, a summary is displayed where characteristics and parameters considered in each methodology are contrasted, as well as the presentation of results and validity specifications. With this it has been found that different methodologies are available to obtain the loss coefficient, through which 
different values are obtained, which may be due mainly to inequalities in the material analyzed and, in the factors, adopted in the investigations. The most studied simple and installed series curves are of $90^{\circ}$ angle with gradual condition and $\mathrm{U}$-shape $\left(180^{\circ}\right)$, respectively. The most suggested works to estimate the coefficient in curves are those of Kirchbach (1929) and Ito (1959, 1960); however, the Idel'chik (1966) manual presents the most extensive information for all the devices studied.

Keywords: Pipe curves, loss of energy, loss coefficient, turbulent flow, flow in pipes, hydraulic modeling.

Recibido: 30/10/2019

Aceptado: $27 / 08 / 2020$

\section{Introducción}

Un desafío que siempre ha tenido el ser humano es el uso eficiente y seguro del recurso hídrico al menor costo posible. Para ello es indispensable explotar y controlar la energía hidráulica, es decir, la energía potencial y la energía cinética que adquiere el fluido (Nasir, 2014; Yuce \& Muratoglu, 2015), estableciéndose una relación directa con la 
Tecnología y

Ciencias $₫$ Agua
2021, Instituto Mexicano de Tecnología del Agua

Open Access bajo la licencia CC BY-NC-SA 4.0

(https://creativecommons.org/licenses/by-nc-sa/4.0/)

segunda ley de la termodinámica (Herwig \& Wenterodt, 2011), donde es esencial cuantificar la energía no aprovechable (pérdida) para conocer la energía hidráulica disponible y utilizarla en los sistemas (Schmandt, Iyer, \& Herwig, 2014), tales como las conducciones en las centrales hidroeléctricas (Elbatran, Yaakob, Ahmed, \& Shabara, 2015); las redes de distribución de agua en poblaciones (Yildirim \& Singh, 2010); los sistemas de riego (Sesma, Molina-Martínez, Cavas-Martínez, \& Fernández-Pacheco, 2015); las líneas de conducción en plantas industriales (Anaya-Durand, Cauich-Segovia, Funabazama-Bárcenas, \& Gracia-Medrano-Bravo, 2014), y las redes de distribución en centrales geotérmica (Maria-Di, 2000), entre otras. Sin embargo, el mismo interés se tiene de conocer la energía disponible en las conducciones con fluidos distintos al agua, los cuales se utilizan en múltiples sistemas de tuberías de plantas industriales (Perumal \& Ganesan, 2016). Dicho interés, que acontece por evaluar las pérdidas de energía en el fluido, se debe a que repercuten principalmente en la selección de la tubería y su diámetro, así como del equipo de bombeo (Yoo \& Singh, 2010).

En la actualidad, el estudio de las pérdidas de energía se divide en pérdidas por fricción y pérdidas locales (Liu, Xue, \& Fan, 2013), teniendo ambas distinto origen, pero igual importancia. En estas últimas se concentra la investigación, en específico en los dispositivos de cambio de dirección conocidos como "curvas", debido a que son muy empleados en los sistemas de tuberías para ajustar el sentido de los conductos (Gontsov, Marinova, \& Tananaev, 1984). No obstante, también son bastante utilizados los otros cambios de dirección llamados "codos", pero merecen una revisión por separado (Kast, 2010; Zmrhal \& Schwarzer, 
Teçnología y

Ciencias $₫$ Agua
2021, Instituto Mexicano de Tecnología del Agua

Open Access bajo la licencia CC BY-NC-SA 4.0

(https://creativecommons.org/licenses/by-nc-sa/4.0/)

2009). Por último, en efecto que las curvas de tuberías son ampliamente utilizadas en los sistemas hidráulicos, se han llevado a cabo diversas investigaciones sobre las pérdidas de energía que éstas generan, las cuales no han cesado en los últimos años, como se puede constatar en los trabajos de: Dang, Yang, Yang e Ishii, (2018); Daneshfaraz, Rezazadehjoudi y Abraham (2018); Reghunathan, Son, Suryan y Kim (2019); Friman y Levy (2019); Tripathi, Portnikov, Levy \& Kalman (2019); Du et al. (2020); Arun, Kumaresh, Natarajan y Kulasekharan (2020); Jia y Yan, (2020). Sin embargo, estas investigaciones recientes se basan en resultados de modelos computacionales, que generalmente se validan contra resultados experimentales de modelos físicos, siendo estos últimos los que analizarán en el presente estudio.

Las investigaciones de las pérdidas de energía en las curvas de tuberías, así como en otros dispositivos, se centralizan particularmente en estimar el coeficiente de pérdidas $(\xi)$; sin embargo, a pesar de numerosos esfuerzos en distintos escenarios, no se ha coincidido satisfactoriamente en los resultados de este coeficiente (Dutta \& Nandi, 2015; Ito, 1960; Kilkovsky, Jegla, \& Stehlik, 2011). Esto conlleva a una incertidumbre en la elección de los datos y metodología a utilizar, adicionando que por lo general los manuales y libros de textos no indican las consideraciones adoptadas en los estudios y para qué tipos de tuberías son aplicables. Portal relevancia, el objetivo de este trabajo es presentar una revisión de las investigaciones y metodologías reportadas en la literatura para estimar el coeficiente $\xi$ en curvas de tuberías bajo flujo turbulento, que permita visualizar detalles importantes de los estudios y 
un contraste de sus características y alcances, a partir de información organizada y homogenizada.

\section{Naturaleza y evaluación de las pérdidas de energía}

Las curvas de tuberías se utilizan en condición brusca y gradual (Figura 1). Cuando un fluido circula por un conducto recto de sección transversal constante y cruza por estos dispositivos, se producen pérdidas de energía en el entorno donde se localizan (Bariviera, Frizzone, \& Rettore, 2014; Fuentes \& Rosales, 2004). Esto es en consecuencia de la modificación del sentido del flujo que altera la distribución de velocidades y presiones, originando que el fluido se separe en la parte interna de la curva y choque sobre la parte externa, lo cual repercute en vórtices y en aumento de la presión; esto conlleva que después de la curva, el movimiento en el flujo ocurra en forma de espiral hasta longitudes aproximadas a 50 veces el diámetro del conducto (Chowdhury, Biswas, Alam, \& Islam, 2016; Ito, 1960; Villegas-León et al., 2016). Sin embargo, cuando la curva se presenta gradualmente (Figura 1b), el fenómeno de perturbación del flujo es similar al provocado en el cambio brusco (Figura 1a), pero éste se 
produce en menor magnitud (Hager, 2010; Kast, 2010; Villegas-León et al., 2016).

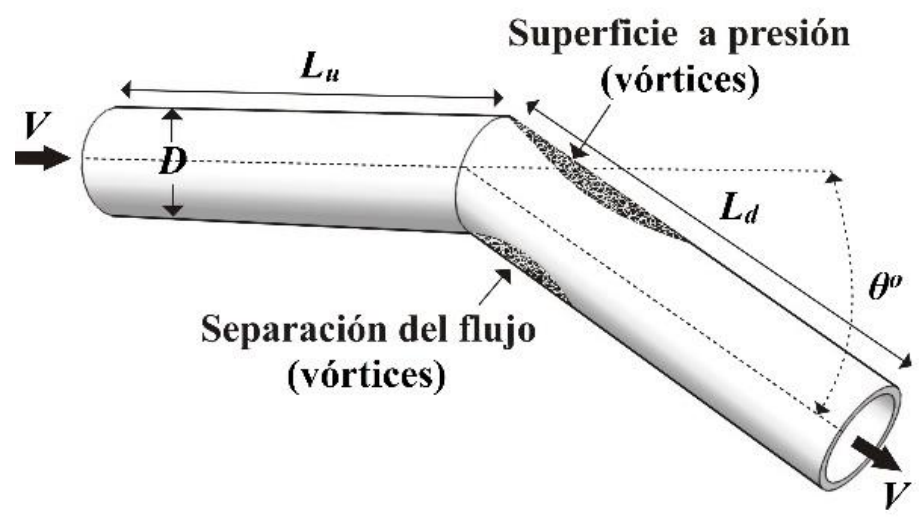

(a)

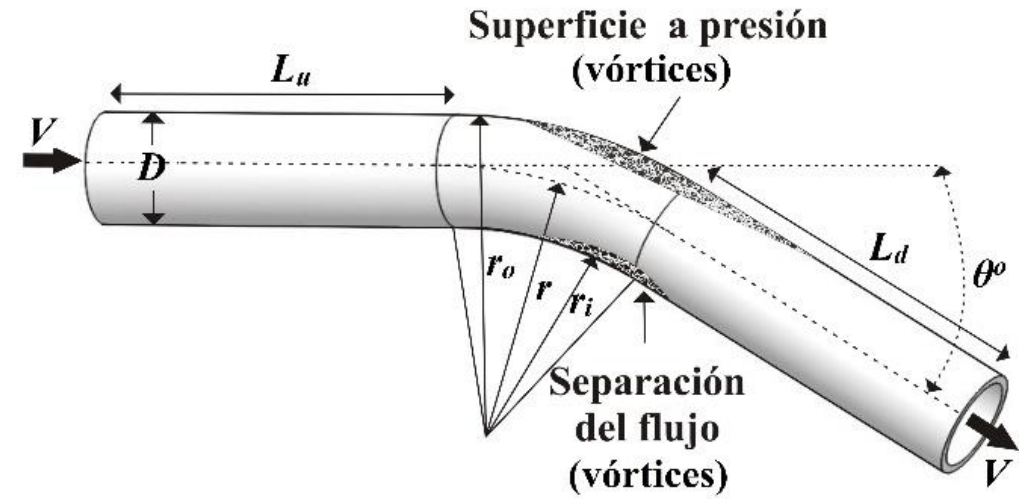

(b)

Figura 1. Comportamiento del flujo circulante en: a) Curva brusca. b) Curva gradual. $\theta$ es el ángulo de deflexión de la curva; $D$ es el diámetro del conducto; $V$ es la velocidad media del flujo; $r$ es el radio de curvatura respecto al eje del dispositivo; $r_{i}$ es el radio de curvatura interno; $r_{o}$ es el radio de curvatura externo del dispositivo; $L_{u}$ y $L_{d}$ son las longitudes de las tuberías aguas arriba y aguas debajo de la curva, respectivamente.

De acuerdo con Beij (1938), Ito (1960), Idel'chik, (1966), Yildirim y Sing (2010), Sotelo (2013), Acero y Rodríguez (2008), USACE (1980) y USBR (1985), entre otros, las pérdidas de locales se determinan mediante la siguiente ecuación general: 
$h_{L}=\xi_{c} \frac{V^{2}}{2 g}$

donde $h_{L}$ es la pérdida de energía hidráulica total $(\mathrm{m})$, que en este trabajo se referirá a las originadas por curvas de tuberías; $g$ es la aceleración de la gravedad $\left(\mathrm{m} / \mathrm{s}^{2}\right) ; V$ es la velocidad media de circulación $(\mathrm{m} / \mathrm{s})$, que en un cambio de dirección es la que se produce aguas arriba del dispositivo; $V^{2} / 2 g$ es la carga por velocidad o energía cinética $(\mathrm{m}) ;$ y $\xi_{C}$ es el coeficiente de pérdidas de la curva (adimensional), el cual es determinado experimentalmente. Para definir el valor del coeficiente de pérdidas de una curva brusca $\left(\xi_{S C}\right)$, por lo general sólo se requiere el ángulo $\theta$, en tanto que para el coeficiente de pérdidas de una curva gradual $\left(\xi_{G C}\right)$, se demanda el ángulo $\theta$ y la relación del radio de la curva con respecto a diámetro del conducto $(r / D)$; sin embargo, en ocasiones ambos coeficientes se relacionan con el número de Reynolds $\left(R_{e}\right)$, el cual se puede expresar para conductos circulares como:

$R_{e}=\frac{\rho V D}{\mu}$

donde $R_{e}$ es adimensional; $\rho$ es la densidad del fluido $\left(\mathrm{kg} / \mathrm{m}^{3}\right) ; V$ es la velocidad media del flujo $(\mathrm{m} / \mathrm{s}) ; D$ es el diámetro del conducto $(\mathrm{m})$, y $\mu$ es la viscosidad dinámica del fluido $(\mathrm{kg} / \mathrm{m} \cdot \mathrm{s})$.

Ahora bien, se dice que la pérdida de energía en curvas es compuesta de la siguiente forma (Beij, 1938; Crane Co., 1982; Ito, 1960): 
$h_{L}=h_{c}+h_{d}+h_{f}$

en la cual $h_{c}$ es la pérdida debida a la alteración del flujo por el tipo de curva que se obtiene de la diferencia de carga de presión del inicio y final del dispositivo; $h_{d}$ es la pérdida excesiva en la longitud de la tubería aguas abajo, con respecto a la pérdida normal por fricción; $h_{f}$ es la pérdida por fricción en la longitud de la curva, la cual puede obtenerse mediante la ecuación de Darcy-Weisbach, que se escribe como sigue:

$h_{f}=f \frac{L}{D} \frac{V^{2}}{2 g}$

donde $L$ es la longitud de la tubería $(\mathrm{m}) ; f$ es un coeficiente de fricción que depende principalmente de $D$, de la rugosidad de la tubería $(\varepsilon)$ y de $R_{e}$; sin embargo, $f$ es ampliamente estudiado y se proporciona en documentos de la temática, tales como USACE (1980), Crane Co. (1982), CFE (1983), USBR (1985) y Saldarriaga (2016), entre otros.

Por otro lado, cabe mencionar que con frecuencia de $h_{L}$ se excluye la pérdida por fricción en la longitud de la curva para tratarse como tal, junto con las pérdidas originadas por tramos rectos de tuberías de un sistema. De esta forma, la pérdida de energía total en una curva quedaría como: 
2021, Instituto Mexicano de Tecnología del Agua

$h_{L}=h_{c}+h_{d}$

Entonces, conociendo la pérdida de energía total, el coeficiente de pérdidas de una curva está dado por la siguiente expresión:

$\xi_{C}=\frac{h_{L}}{V^{2} / 2 g}$

Finalmente, es importante verificar si en $h_{L}$ se considera o no la pérdida por fricción en el trayecto de la curva, ya que ello repercute en el valor del coeficiente de pérdidas. En el siguiente apartado se presentan resultados de investigaciones y metodologías reportadas en la literatura para estimar el coeficiente de pérdidas de curvas de tuberías.

\section{Estimación del coeficiente de pérdidas}

A continuación se expone un análisis de las investigaciones y metodologías revisadas en la literatura sobre las pérdidas de energía y el coeficiente de pérdidas de curvas de tuberías, donde se analizan cinco casos específicos: el caso de dispositivo simple (Figura 1) y otros cuatro 
casos de estructuras conformadas con dispositivos simples en serie (uno seguido de otro), los cuales se clasifican como forma-L, forma-S, formaU y forma-Z (Figura 2). En los escenarios de estudio mencionados, las longitudes de tuberías se tratarán como proporcionales a sus diámetros internos $(D)$ o al radio de la curva $(r)$. Algunos casos que autores solo mencionan, como el diámetro nominal de las tuberías $(D n)$. Los valores de $D$ serán estimados de los valores de $D n$ indicados en Shames (1995), para las tuberías de acero al carbón, aleaciones de acero y acero inoxidable.
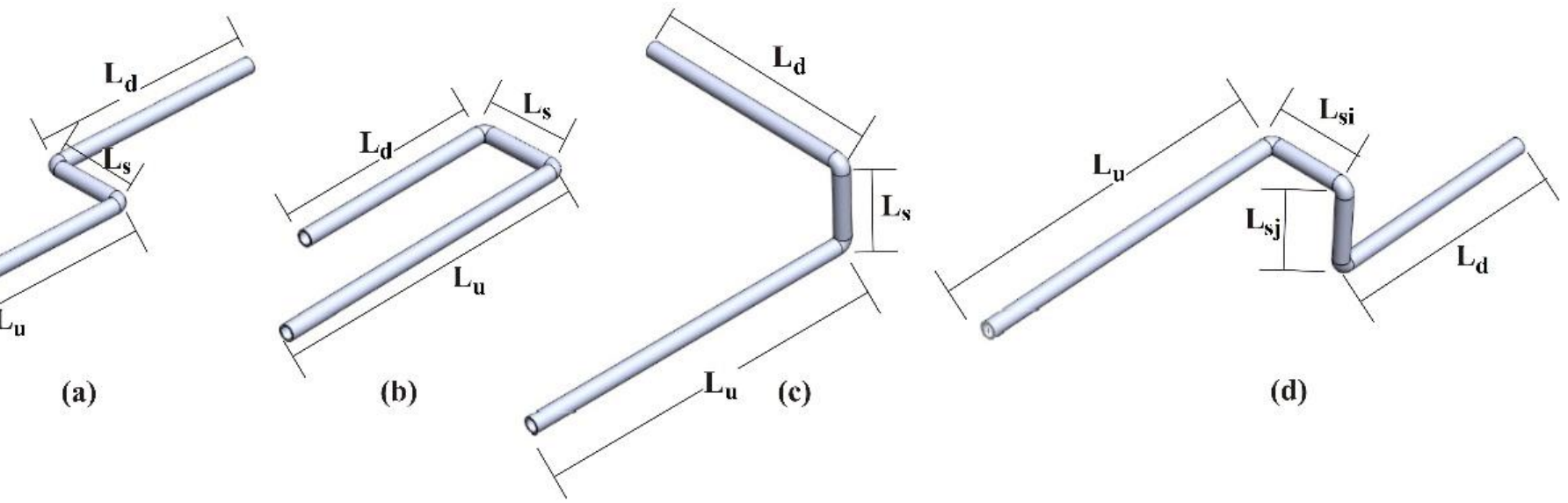

(d)

Figura 2. Casos de estudio de estructuras con curvas en serie: a) forma-Z, b) forma- $U$, c) forma-L y d) forma-S. $L_{u}$ y $L_{d}$ es la longitud de la tubería aguas arriba y aguas abajo del dispositivo, respectivamente; $L_{s}, L_{s i}$ y $L_{s j}$ son longitudes de separación entre dos dispositivos colocados en serie. 
Ciencias $₫$ Agua
2021, Instituto Mexicano de Tecnología del Agua

Open Access bajo la licencia CC BY-NC-SA 4.0

(https://creativecommons.org/licenses/by-nc-sa/4.0/)

Uno de los trabajos ampliamente citados es el de Beij (1938), quien realizó una investigación experimental de la pérdida de presión causada por curvas graduales de acero $\operatorname{con} \theta=90^{\circ}, D n=10.16 \mathrm{~cm}$ y $D=10.23$ $\mathrm{cm}$; sin embargo, también se obtuvieron valores de $h_{f}$ en los tramos de tuberías $L_{u}=48.15 D$ y $L_{d}=168.12 D$. Para ello, se realizaron ensayos con agua en nueve curvas horizontales de $9.92 \leq r \leq 204.2(\mathrm{~cm})$. Por medio de una serie de piezómetros se evaluaron diferencias de alturas de presión en distintos puntos del sistema tanto aguas arriba como aguas abajo del dispositivo, mientras que la carga de velocidad se estimó a partir del caudal aforado y el área de la sección transversal del conducto. Con ello se obtuvieron resultados del coeficiente de las curvas graduales $\left(\xi_{G C}\right)$ para relaciones de $0.97 \leq r / D \leq 19.96$ y valores aproximados de $0.23 \times 10^{5} \leq$ $R_{e} \leq 3.4 \times 10^{5}$. Los valores del $\xi_{G C}$ no presentaron una tendencia definida para valores del $R_{e}$, por lo cual también se presentan valores promedio del $\xi_{G C}$ sólo en función de $r / D$. Estos resultados se presentan en un gráfico, donde se muestra que el coeficiente disminuye de 0.37 a 0.16 y después incrementa de 0.16 a 0.41 , cuando la relación $r / D$ incrementa de 1 a 3.5 y de 3.5 a 20, respectivamente; por lo tanto, el valor mínimo y máximo de $\xi_{G C}$ es 0.16 y 0.41 , cuando $r$ vale 3.5 y $40 \mathrm{~cm}$, respectivamente. Estos valores fueron comparados en otro gráfico ante los datos obtenidos experimentalmente por Hofmann (1929), en curvas lisas y rugosas de $D$ $=4.32 \mathrm{~cm}$; Davis (1911), en curvas de $D=5.08 \mathrm{~cm}$; Balch (1913), en curvas de $D=7.62 \mathrm{~cm}$; Vogel (1933), en curvas de $D=15.24,20.32$ y $25.40 \mathrm{~cm}$; y por Brightmore (1907), en curvas de $D=7.62$ y $10.16 \mathrm{~cm}$. En esta comparación de resultados, los valores del $\xi_{G C}$ no son congruentes entre un autor y otro. No obstante, el valor máximo del $\xi_{G C}=0.58$ y es 
2021, Instituto Mexicano de Tecnología del Agua

Open Access bajo la licencia CC BY-NC-SA 4.0

(https://creativecommons.org/licenses/by-nc-sa/4.0/)

dado por Balch (1913) con la relación $r / D=15$, entretanto que el valor mínimo del $\xi_{G C}=0.08$ y se obtiene de Hofmann (1929) en tuberías lisas, con la relación $r / D=7$.

Con la finalidad de evaluar el comportamiento de la pérdida de energía en curvas graduales de latón fundido, Ito (1960) realizó experimentos en dispositivos con aproximadamente valores de $D=3.5$ $\mathrm{cm}$ y de $\theta=45,90$ y $180^{\circ}$. La curva de $180^{\circ}$ es una estructura en forma$\mathrm{U}$, la cual se conforma por dos curvas en serie de $90^{\circ}$ de deflexión, que producen una $L_{s}=2 r$. Las curvas de $45^{\circ}$ tuvieron valores de $r=6.42$, 11.3 y $25.54 \mathrm{~cm}$; las curvas de $90^{\circ}$ presentaron valores de $r=3.52,6.38$ y $11.42 \mathrm{~cm}$; y las curvas de $180^{\circ}$ fueron con valores de $r=6.39$ y 11.32 $\mathrm{cm}$. A los dispositivos estudiados se les acoplaron tuberías de cobre aguas arriba y aguas abajo, con $L_{u}=153.8 D$ y $L_{d}>71.6 D$. Para la práctica se utilizó agua a carga constante, así como un sistema de manómetros para estimar las alturas de presión en cinco puntos aguas arriba de la curva y en ocho puntos aguas debajo de ella. En cada punto se evaluaron las alturas de presión en el lado derecho e izquierdo sobre el eje de la sección transversal, así como en la parte superior e inferior de la misma, donde se evaluó $h_{L}=h_{c}+h_{d}$. Considerando lo anterior, se obtuvieron valores del $\xi_{G C}$ para un rango de $0.2 \times 10^{5} \leq R_{e} \leq 4.0 \times 10^{5}$, evaluando $h_{d}$ en valores de $L_{d}=0,9 D, 18 D$ y $>50 D$; los resultados evidenciaron que el coeficiente disminuye conforme incrementa $R_{e}$. A partir de ello, el autor propuso ecuaciones para estimar el coeficiente de pérdidas, las cuales también se recomiendan en Lourenco y Xin (2017), mismas que se exponen a continuación: 


\section{1, Instituto Mexicano de Tecnología del Agua}

Para $0.25 R_{e}(r / D)^{-2}<91$

$\xi=0.01746 K f \theta(r / D)$

Para $0.25 R_{e}(r / D)^{-2}>91$

$\xi=0.004314 K \theta R_{e}^{-0.17}(r / D)^{0.84}$

donde $\xi$ es el coeficiente de pérdidas de una curva gradual $\left(\xi_{G C}\right)$ y una estructura en forma-U $\left(\xi_{U S}\right) ; f$ se especifica en Ito (1959); $K$ es un coeficiente adimensional que depende de $\theta$ y $r / D$, el cual se estima mediante un gráfico o con ecuaciones sugeridas por el autor, las cuales se pueden escribir de la siguiente forma:

Si $\theta=45^{\circ}$

$K=1+5.126(r / D)^{-1.47}$

Si $\theta=90^{\circ}$ y $2(r / D)<19.7$

$K=0.95+4.421(r / D)^{-1.96}$ 
Si $\theta=90^{\circ}$ y $2(r / D)>19.7$

$K=1.0$

Si $\theta=180^{\circ}$

$K=1+5.056(r / D)^{-4.52}$

Cabe destacarque en White (2008) se sugiere estimar el coeficiente de pérdidas de una curva de $90^{\circ}$ mediante la ecuación para $0.25 R_{e}(r / D)^{-}$ $2>91$. Por otro lado, en la comparación de los resultados para las curvas de $45^{\circ}, 90^{\circ}$ y en forma-U se manifestó buena correlación entre los coeficientes experimentales obtenidos y los estimados con la ecuación (3), cuando $L_{d}>50 \mathrm{D}$. Sin embargo, entre diversas presentaciones gráficas de los resultados se destacan los siguientes casos:

a) Los resultados del $\xi_{G C}$ de las curvas de $45^{\circ}$ son comparados con los valores estimados mediante la Ecuación (3), la ecuación de Weisbach (1855) y la ecuación de Richter (1930). Los resultados de la ecuación de Weisbach (1855) se apegaron a los obtenidos experimentalmente con $L_{d}=18 D$, mientras que los determinados mediante la ecuación de Richter (1930) se inclinaron a los investigados con $L_{d}=0$.

b) No obstante, los valores obtenidos para el $\xi_{G C}$ de las curvas de $90^{\circ}$ fueron comparados contra los valores calculados con la Ecuación (3) y las ecuaciones propuestas por Weisbach (1855), Richter (1930), Pigott 
Ciencias $₫$ Agua
2021, Instituto Mexicano de Tecnología del Agua

Open Access bajo la licencia CC BY-NC-SA 4.0

(https://creativecommons.org/licenses/by-nc-sa/4.0/)

(1950) y Pigott (1957). En esta parte, los valores de $\xi_{G C}$ estimados con la ecuación de Weisbach (1855) fueron más congruentes a los examinados con $L_{d}>50 D$; los determinados con la ecuación de Pigott (1957) resultaron mayores que todos; y los obtenidos con las ecuaciones de Richter (1930) y Pigott (1950), se inclinaron más a los coeficientes con bajos valores de $L_{d}$.

c) En el caso de las curvas en forma-U se utilizó el mismo criterio de comparación que en las curvas de $45^{\circ}$. Aquí, los valores del $\xi_{U S}$ estimados mediante la ecuación de Weisbach (1855) fueron mayores a todos los casos, mientras que los determinados mediante la ecuación de Richter (1930) se sesgaron más sobre valores bajos de $L_{d}$.

d) Por otro lado, en otro gráfico se exponen los resultados experimentales del coeficiente de pérdidas en curvas lisas con $R_{e}=2 \times 10^{5}$ y ángulos de $45^{\circ}, 90^{\circ}$ y en forma-U, en función de $r / D$ de 1 a 15; el gráfico también se indica en White (2008) para estimar el coeficiente de pérdidas en mención. Estos datos son confrontados ante los valores obtenidos mediante la Ecuación (3), la propuesta por Hofmann (1929) y la sugerida por Wasielewski (1932). Los resultados experimentales y analíticos del autor presentaron buena correlación con los dados por Wasielewski (1932), cuando $\theta=45^{\circ}$, y también ante los resultados estimados por Hofmann (1929), cuando $\theta=90^{\circ}$.

e) Por último, se exponen resultados experimentales del coeficiente de pérdidas, que fueron estudiados en 10 curvas de cobre con radio largo, $\operatorname{con} \theta=45,90,135$ y $180^{\circ}$ (forma-U), así como con $r / D$ de 5.2 a 108; se destaca que previamente se han reportado en Ito (1956). Dichos 
2021, Instituto Mexicano de Tecnología del Agua

Open Access bajo la licencia CC BY-NC-SA 4.0

(https://creativecommons.org/licenses/by-nc-sa/4.0/)

resultados indicaron que conforme incrementa la relación $r / D$ para un mismo valor de $\theta$, también aumenta $h_{c}$ y decrece $h_{d}$. En un gráfico se exponen los resultados de los coeficientes $\xi_{G C}$ y $\xi_{U S}$ para un rango de 0.1 × $10^{5} \leq R_{e} \leq 3.0 \times 10^{5}$, donde se muestra que dichos coeficientes se reducen conforme se eleva el $R_{e}$. Además, se ilustra que los valores mínimos de los coeficientes son obtenidos $\operatorname{con} \theta=90^{\circ}$ y $r / D=7.6$; $\sin$ embargo, los valores máximos son dados $\operatorname{con} \theta=45^{\circ}$ y $r / D=108$.

Respecto a curvas comerciales de PVC, Chen-Tzu (1969) llevó a cabo un estudio experimental sobre el comportamiento del flujo y las pérdidas de energía en curvas $\operatorname{con} \theta=90^{\circ}$ y $r / D=1.59$. Los dispositivos utilizados fueron de dos pulgadas con $D=5.25 \mathrm{~cm}$, los cuales se acoplaron a tuberías con las mismas características, y de longitudes $L_{u}=$ $100 D$ y $L_{d}>36 D$. Las pruebas se hicieron en curvas de sentido horizontal y vertical, utilizando distintos flujos de agua, con temperatura aproximada a $21.1{ }^{\circ} \mathrm{C}$, que produjeron $R_{e}$ de $0.282 \times 10^{5}$ a $1.86 \times 10^{5}$. Mediante un sistema de piezómetros se evaluaron las presiones a cada $90^{\circ}$ en la circunferencia de sección transversal, permitiendo obtener las alturas de presión en el lado izquierdo y derecho del eje de la conducción, y en la parte superior e inferior del mismo; dichas presiones se estimaron en el centro del ángulo de la curva y hasta distancias de $30 \mathrm{D}$ aguas arriba del dispositivo y $36 \mathrm{D}$ aguas abajo. Entre otros resultados, se presenta un gráfico del $\xi_{G C}$ para el sentido horizontal y vertical, conforme a la variación de $R_{e}$. En el gráfico se muestra que, en ambos casos, el $\xi_{G C}$ se reduce a medida que incrementa el $R_{e}$, pero con diferente comportamiento. En los rangos aproximados a $R_{e}<0.57 \times 10^{5}$ y $R_{e}>1.4 \times 10^{5}$, el $\xi_{G C}$ resultó ligeramente mayor en la curva vertical, mientras que en el rango de 0.57 
2021, Instituto Mexicano de Tecnología del Agua

Open Access bajo la licencia CC BY-NC-SA 4.0

(https://creativecommons.org/licenses/by-nc-sa/4.0/)

x $10^{5}<R_{e}<1.4 \times 10^{5}$, el $\xi_{G C}$ resultó levemente superior en el caso horizontal; por ende, el $\xi_{G C}$ resulta igual para la curva horizontal y vertical cuando $R_{e} \approx 0.57 \times 10^{5}$ y $1.4 \times 10^{5}$, presentando valores de 0.24 y 1.8 , respectivamente. Por último, los valores máximos de $\xi_{G C}$ en la curva horizontal y vertical fueron de 0.35 y 0.38 , en tanto que los valores mínimos fueron de 0.16 y 0.18 , respectivamente, los cuales se presentaron en los valores mínimos y máximos de $R_{e}$.

Más de una década después, Turian, Hsu y Selim (1983) presentan una investigación sobre del coeficiente de pérdidas para algunos dispositivos y válvulas de tuberías de acero, donde se utilizaron flujos con sólidos en suspensión. Dicha investigación se dice que fue realizada previamente por Hsu (1981), en la cual se obtuvieron valores del coeficiente de pérdidas para curvas bruscas y graduales de acero con $D$ $=2.54$ y $5.08 \mathrm{~cm}$, utilizando distintos concentrados de perlas de vidrio del 0 al $50 \%$ del peso. Las curvas bruscas fueron de $90^{\circ}$ de deflexión, mientras que las graduales tuvieron 45,90 y $180^{\circ}$ (forma- $U, L_{s}=0$ ). Las curvas de $45^{\circ}$ y en forma-U presentaron un $r=3.81 \mathrm{~cm}$ y $5.72 \mathrm{~cm}$, en el $D$ menor y mayor, respectivamente. Sin embargo, las curvas graduales de $90^{\circ}$ fueron de $3.81,11.43,21.59$ y $31.75 \mathrm{~cm}$ de radio para el $D$ menor, así como $5.72,22.86,43.18$ y $63.50 \mathrm{~cm}$ de radio para el $D$ mayor. Los resultados se exponen en gráficos y tablas, de donde se resalta lo siguiente:

a) El porcentaje de concentración de sólidos no incide de modo significativo en el valor del coeficiente de pérdidas en todas las curvas estudiadas, tendiendo a promediarse en un comportamiento asintótico. 
Tecnología y



2021, Instituto Mexicano de Tecnología del Agua

Open Access bajo la licencia CC BY-NC-SA 4.0

(https://creativecommons.org/licenses/by-nc-sa/4.0/)

b) El coeficiente de pérdidas para los dispositivos con $D=2.54 \mathrm{~cm}$ se presentan en función del $R_{e}$ de 0.1 a $0.8 \times 10^{5}$, donde el coeficiente de pérdidas tiende a ser asintótico a partir de $R_{e} \geq 0.25 \times 10^{5}$.

c) Los resultados del coeficiente en los dispositivos con $D=5.08$ se indicaron con $R_{e}$ de 0.2 a $1.7 \times 10^{5}$, en los cuales se mostró que dicho coeficiente sigue un comportamiento asintótico en $R_{e} \geq 0.4 \times 10^{5}$.

d) En una tabla se presentan valores promedio del coeficiente de pérdidas para los dispositivos con $D=2.54 \mathrm{~cm}$ y $5.08 \mathrm{~cm}$, bajo flujos con $R_{e} \geq 0.25$ x $10^{5}$ y $R_{e} \geq 0.4 \times 10^{5}$, respectivamente. Los coeficientes de los dispositivos de $D$ menor fueron de 0.94 en la curva de $45^{\circ} ; 1.63$ en la curva brusca de $90^{\circ}$; el mínimo de 0.80 y el máximo de 1.27 en curvas graduales de $90^{\circ}$ con $r=21.59$ y $3.81 \mathrm{~cm}$, respectivamente; y 0.90 en curvas en forma-U. No obstante, dichos coeficientes en los dispositivos de $D$ mayor resultaron de 0.73 en curva de $45^{\circ} ; 1.91$ en la curva brusca de 900; el mínimo de 0.60 y el máximo de 1.13 en curvas graduales de $90^{\circ}$ con $r=22.86$ y $43.18 \mathrm{~cm}$, respectivamente, y 0.92 en curvas en formaU.

Además de mostrar resultados experimentales, Turian et al. (1983) menciona que el coeficiente de pérdidas en curvas puede obtenerse mediante las ecuaciones de Ito (1959) e Ito (1960) para $0.25 R_{e}(r / D)^{-2}<$ 91 y $0.25 R_{e}(r / D)^{-2}>91$ (expuestas anteriormente), y también utilizando una tabla indicada en Crane Co. (1982). Se contrastan los resultados experimentales sólo contra los de Crane Co. (1982), donde se encontraron diferencias notables para los dos tipos de diámetros; siempre resultaron mayores los coeficientes obtenidos en la investigación, excepto 
para los de forma-U, que resultaron menores a los dados en la literatura. En dicha comparación de los dispositivos con $D=2.54 \mathrm{~cm}$, las diferencias mínima y máxima de los coeficientes fueron de 0.25 en la curva en forma$U$ y de 0.67 en la curva de $90^{\circ}$ con $r=11.43 \mathrm{~cm}$, respectivamente; entretanto, dichas diferencias en los dispositivos con $D=5.08 \mathrm{~cm}$ fueron de 0.03 en la curva en forma-U y de 0.77 en la curva brusca de $90^{\circ}$.

Por otra parte, Gontsov et al. (1984) realizaron una investigación en laboratorio sobre el comportamiento del flujo y de la pérdida de energía en una curva horizontal $\operatorname{con} \theta=90^{\circ}$, utilizando flujo isotérmico de aire. El modelo se construyó a base de vidrio orgánico de $0.6 \mathrm{~cm}$ de espesor, con $D=20.6 \mathrm{~cm}, r / D=1, L_{u}=35 D$ y $L_{d}=25 D$. Las presiones en el flujo y sobre las paredes se evaluaron mediante manómetros diferenciales con alcohol, considerando valores del $R_{e}$ de 0.9 a $4.0 \times 10^{5}$. Los resultados se indican mediante gráficos, sin embargo, también se manifiestan cuatro ecuaciones para obtener el $\xi_{G C}$. La primera es una ecuación que se le atribuye a D. Al'tshul, y se escribe de la forma siguiente:

$\xi_{G C}=\left[0.2+0.001(100 f)^{8}\right](r / D)^{-0.5}$

La segunda ecuación expuesta es la reportada por Ito (1960) y citada previamente en la Ecuación (8). Las dos ecuaciones restantes son obtenidas por los autores para curvas graduales y lisas, a partir de sus propias investigaciones y los resultados de Ito (1960). La tercera ecuación 
es sugerida para $0.2 \times 10^{5}<R_{e}<4.0 \times 10^{5}$, la cual se expone a continuación:

$$
\xi_{G C}=1.45 R_{e}^{-0.15}(r / D)^{-0.3}
$$

La cuarta ecuación es recomendada para $R_{e} \geq 4.0 \times 10^{5}$, donde el $\xi_{G C}$ es independiente del régimen del flujo. Dicha ecuación se escribe como:

$$
\xi_{G C}=0.21(r / D)^{-0.3}
$$

Finalmente, cabe resaltar que los valores experimentales obtenidos del coeficiente de pérdidas presentaron buena correlación con los resultados logrados en Ito (1960), cuando se tiene el rango de $1 \leq r / D \leq$ 3.

Con base en el análisis de la información experimental reportada en la literatura, en Villegas-León et al. (2016) se proponen ecuaciones para el $\xi_{S C}$ y $\xi_{G C}$, las cuales generan valores promedio a partir de los obtenidos para el coeficiente con las metodologías consideradas. En el caso de las curvas bruscas, se seleccionaron las metodologías indicadas en CFE (1983), Miller (1990), Sotelo (2013) y USACE (1980), sobre las cuales se obtuvo la siguiente expresión:

$\xi_{S C}=\frac{0.0031960558+0.0030444516(\theta)}{1-0.014390831(\theta)+0.00006719314(\theta)^{2}}$ 
Se indica que la ecuación anterior es válida para valores de $0<\theta \leq$ $90^{\circ}$. Por otro lado, sobre curvas graduales se trabajaron las metodologías de CFE (1983), Miller (1990), Sotelo (2013), SARH (1984), USACE (1980) y USBR (1985), de cuales se obtuvo la ecuación siguiente:

$\xi_{G C}=\frac{A+C \theta}{B}$

donde $A, B$ y $C$ son coeficientes que dependen de la relación $r / D$, y se determinan mediante las siguientes ecuaciones:

$A=-0.0573379+0.00496834(r / D)-0.00001716(r / D)^{3}+\frac{0.07867083}{\left(r^{0} / D\right)^{0.5}}-\frac{0.066727}{\left.e^{(r / D}\right)}$

$B=0.20495202+0.05446522(r / D)-0.08723377(r / D)^{0.5} \ln (r / D)-$ $\frac{0.45002930 \ln (r / D)}{(r / D)}-\frac{0.25130468}{(r / D)^{2}}$

$C=-0.01383436-0.01385106\left({ }^{r} / D\right)+0.00051449(r / D)^{2}+$

$0.04504019 \ln (r / D)+\frac{0.08991395}{\left.e^{(r / D}\right)}$

Se establece que estas ecuaciones para la condición gradual son válidas bajo los rangos de $1 \leq r / D \leq 10$ y $5 \leq \theta \leq 90^{\circ}$. 
Ahora, en los párrafos restantes se presentan diversos métodos para estimar el coeficiente de pérdidas de curvas de tuberías, los cuales son sugeridos en manuales y libros de la temática, basándose en experiencias propias del autor $y / 0$ en resultados de otros investigadores, sin exponer detalles de los desarrollos experimentales.

En el documento de Gibson (1930) se dice que los primeros experimentos de pérdida de energía en curvas de tuberías fueron realizados por Weisbach (1855), utilizando tuberías de acero con $D=$ $3.175 \mathrm{~cm}$. A partir de dichos experimentos y otros más, propuso las siguientes ecuaciones para las curvas bruscas y graduales:

$\xi_{S C}=0.946 \sin ^{2} \frac{\theta}{2}+2.05 \sin ^{4} \frac{\theta}{2}$

$\xi_{G C}=\left[0.000222+0.000276(r / D)^{-3.5}\right] \theta$

La primera ecuación que es para el coeficiente de curvas bruscas también es sugerida para codos de la misma condición. Además, se presentan valores tabulados del $\xi_{G C}$ derivados de resultados de Brightmore (1907) para curvas de hierro fundido con $D=7.62$ y 10.16 $\mathrm{cm}, r / D$ de 2 a 10, y bajo velocidades de 1.52 a 3.05 m/s. Dichos resultados no mostraron un comportamiento bien definido ante la variación de $r / D$ y la velocidad del flujo; sin embargo, para los dos diámetros estudiados, los valores mínimos y máximos se puede asumir que se presentan en $r / D=10$ y 2 , respectivamente. En las curvas con $D$ 
Tecnología y

Ciencias $₫$ Agua
2021, Instituto Mexicano de Tecnología del Agua

Open Access bajo la licencia CC BY-NC-SA 4.0

(https://creativecommons.org/licenses/by-nc-sa/4.0/)

$=7.62 \mathrm{~cm}$, el coeficiente menor y mayor fue aproximadamente de 0.22 y 0.43 , mientras que para $D=10.16 \mathrm{~cm}$ resultaron de 0.23 y 0.38 , respectivamente. Finalmente, también se presentan valores del coeficiente de pérdidas en curvas, estimados a partir de la investigación de Schoder (1908) sobre dispositivos de hierro forjado, que presentaron un $D n=6$ pulgadas, $D$ estimado de $15.4 \mathrm{~cm}$ y relaciones $r / D$ de 1.34 a 20. Los valores son expuestos en una tabla en función de $r / D$ y de tres velocidades distintas: 1.52, 3.05 y 4.88 m/s. En este caso, el comportamiento del coeficiente resultó oscilatorio, pero tomando en cuenta el promedio de las velocidades, presentó el valor mínimo de 0.27 en $r / D=5$ y 10 , mientras que el valor máximo fue de 0.45 en $r / D=1.34$.

Ahora, Daugherty e Ingersoll (1954) exponen que en curvas graduales $\operatorname{con} \theta=90^{\circ}$, el coeficiente de pérdidas se obtiene mediante la ecuación de Pigott (1950), la cual se escribe de la siguiente forma:

$\xi_{G C}=0.106(r / D)^{-2.5}+2 f^{2.5}$

Debido que el valor de $R_{e}$ incide en $f$, implica que también influya en $\xi_{G C}$. Por último, se indica que en curvas con $\theta \neq 90^{\circ}$, el valor de $\xi_{G C}$ es aproximadamente proporcional al valor de $\theta$. Es decir, que en una curva de 45 y $180^{\circ}$, el valor de $\xi_{G C}$ es el 50 y $200 \%$, respectivamente, sobre el valor obtenido con la ecuación anterior.

En el manual de Idel'chik (1966) se presenta amplia información sobre las pérdidas de energía en los dispositivos bajo estudio, donde se establece que la pérdida total es conformada por la pérdida local y la 
pérdida por fricción a lo largo de las curvas. Sobre las pérdidas por fricción, el autor proporciona una serie de ecuaciones, tablas y gráficos para obtener el coeficiente $f$, sin embargo, no se abundará en ello. Respecto a las pérdidas locales (Ecuación (1)), se presenta una gama de ecuaciones, tablas y gráficos, que se obtienen a partir de resultados experimentales de diversos autores. Acorde con dicha información se clasificaron los casos siguientes para curvas simples y curvas en serie:

a) Si las curvas son simples, graduales y con superficies lisas $(\varepsilon \approx 0)$, que cumplen con $0<\theta \leq 180^{\circ}, r / D \geq 0.5$ y $R_{e} \geq 2 \times 10^{5}$, el coeficiente de pérdidas se obtiene bajo la ecuación de Abramovich (1935):

$\xi_{G C}=\delta \alpha$

en la cual $\delta$ y $\alpha$ son coeficientes que dependen de $\theta$ y $r / D$, respectivamente, los cuales se estiman de gráficos y tablas establecidas por Nekrasov (1954), a partir de las investigaciones de Idel'chik (1953), Evdomikov (1940) y propias. Ahí, el coeficiente $\delta$ incrementa de 0 a 1.4, cuando $\theta$ aumenta de 0 a $180^{\circ}$, mientras que $\alpha$ decrece de 1.18 a 0.17 , cuando $r / D$ incrementa de 0.5 a 1.5 , respectivamente. Sin embargo, también se establecen las siguientes ecuaciones para estimar los coeficientes $\delta$ y $\alpha$ :

Si $\theta \leq 70^{\circ}$ : 
2021, Instituto Mexicano de Tecnología del Agua

Open Access bajo la licencia CCBY-NC-SA 4.0

(https://creativecommons.org/licenses/by-nc-sa/4.0/)

$\delta=0.9 \sin \theta$

Si $\theta=90^{\circ}$ :

$\delta=1.0$

Si $\theta \geq 100^{\circ}$ :

$\delta=0.7+0.0039 \theta$

Si $0.5 \leq r / D \leq 1.0$ :

$\alpha=0.21(r / D)^{-2.5}$

Si $r / D>1.0$ :

$\alpha=0.21(r / D)^{-0.5}$

b) Cuando las curvas son simples y graduales, con superficies rugosas $(\varepsilon>$ $0)$, $r$ corto, $0<\theta \leq 180^{\circ}$ y $0.5<r / D \leq 1.5$, para conducir flujos con $R_{e}$ $\geq 0.03 \times 10^{5}$, el coeficiente se estima con la siguiente ecuación: 
2021, Instituto Mexicano de Tecnología del Agua

Open Access bajo la licencia CC BY-NC-SA 4.0

(https://creativecommons.org/licenses/by-nc-sa/4.0/)

$\xi_{G C}=\delta \alpha \lambda_{1} \lambda_{2}$

donde los coeficientes $\delta$ y $\alpha$ se determinan conforme a lo indicado en a); $\lambda_{1}$ y $\lambda_{2}$ son coeficientes que se estiman mediante tablas en función de $R_{e}$, $r / D$ y $\varepsilon / D . \lambda_{1}$ y $\lambda_{2}$ pueden incrementar de $45 f$ a 1.0 y 1.0 a 2.0, respectivamente, a medida que también aumenta el valor de los parámetros que los rigen.

c) Si las curvas son simples y graduales, con superficies rugosas $(\varepsilon>0), r$ largo, $0<\theta \leq 180^{\circ}$ y $1.5<r / D \leq 50$, donde se presentan flujos con $R_{e} \geq$ $0.03 \times 10^{5}$, el $\xi_{G C}$ también se determina con la Ecuación (30), pero ahora el autor proporciona nuevas tablas y gráficos para estimarlos coeficientes $\delta, \alpha, \lambda_{1}$ y $\lambda_{2}$. Ahí el coeficiente $\delta$ puede incrementar de 0 a 1.4, cuando $\theta$ aumenta de 0 a $180^{\circ}$, mientras que $\alpha$ decrece de 0.21 a 0.30 , cuando $r / D$ incrementa de 1 a 50, respectivamente. Sin embargo, los coeficientes $\lambda_{1}$ y $\lambda_{2}$ incrementan de $64 f$ a 1.0 y 1.0 a 2.0 , respectivamente, a medida que también aumenta el valor de $R_{e}$ y $\varepsilon / D$.

d) En el caso de curvas simples y graduales, con superficies lisas $(\varepsilon \approx 0), r$ largo, $0<\theta \leq 90^{\circ}$ y $r / D \gg 1.5$, para conducir flujos con $50<R_{e}<0.2 \mathrm{x}$ $10^{5}$, el coeficiente de pérdida local incluye el coeficiente de resistencia a la fricción total de la curva, por lo cual no debe evaluarse por separado. En este caso, el $\xi_{G C}$ debe estimarse bajo la ecuación de Aronov (1950), que se basa en los resultados de Adler (1934), White (1929) y propios. Dicha ecuación se escribe de la siguiente manera: 
Tecnología y

Ciencias $\stackrel{\unlhd}{\Im}$ Agua
2021, Instituto Mexicano de Tecnología del Agua

Open Access bajo la licencia CC BY-NC-SA 4.0

(https://creativecommons.org/licenses/by-nc-sa/4.0/)

$\xi_{G C}=0.0175 f(r / D) \theta$

donde $f$ se obtiene a través de tablas y gráficos en función de $r / D$ y $R_{e}$. En dichos datos, el valor del $f$ disminuye a medida que incrementan sus parámetros gobernantes. El valor máximo de $f$ es de 0.34 , cuando $r / D=$ 3.1 y $R_{e}=400$, mientras que su valor mínimo es de a 0.029, cuando $r / D$ $=100$ y $R_{e}=0.2 \times 10^{5}$. Por otro lado, también se proporcionan tres ecuaciones para estimar $f$, las cuales fueron obtenidas por Aronov (1950), a partir de los resultados de las investigaciones antes mencionadas, que al sustituirlas en la ecuación anterior se produce lo siguiente:

Si $50<R_{e}(D / 2 r)^{0.5} \leq 600$ :

$\xi_{G C}=0.31 \theta R_{e}^{-0.65}(r / D)^{0.825}$

Si $600<R_{e}(D / 2 r)^{0.5} \leq 1400$ :

$\xi_{G C}=0.1557 \theta R_{e}^{-0.55}(r / D)^{0.775}$

Si $1400<R_{e}(D / 2 r)^{0.5} \leq 5000$ :

$\xi_{G C}=0.0723 \theta R_{e}^{-0.45}(r / D)^{0.725}$ 
e) Por otra parte, se presenta un gráfico para estimar el coeficiente de pérdidas en curvas simples de condición brusca y con $90^{\circ}$ de deflexión, el cual es aplicable tanto para curvas con $r_{i}=0$ y $r_{o} \geq 0$ como en curvas con $r_{i} \geq 0$ y $r_{o}=0$. En el caso que $r_{o}=0$, el $\xi_{S C}$ resulta mayor que cuando $r_{i}$ $=0$. Los valores mínimos y máximos aproximados que dicho coeficiente puede tomar con $r_{o}=0$ son de 1.1 y 1.38 , cuando $r_{i} / D$ vale 1.1 y 2.1 , respectivamente; mientras que con $r_{i}=0$ puede tomar los valores de 0.18 y 1.1 , cuando $r_{o} / D$ vale 1.2 y 0 , respectivamente.

f) Cuando se tiene una curva o codo simple, con condición brusca $(r / D=$ $0)$, superficies lisas $(\varepsilon \approx 0)$ y $0<\theta \leq 180^{\circ}$, que conduce flujos con $R_{e} \geq$ $0.4 \times 10^{5}$, el coeficiente de pérdidas debe determinarse mediante la ecuación de Abramovich (1935), la cual se escribe como sigue:

$\xi_{S C}=\delta_{1} \delta_{2}$

donde $\delta_{1}$ y $\delta_{2}$ son coeficientes que dependen de $\theta ; \delta_{1}$ se obtiene de tablas y gráficos con datos de Richter (1930), Richter (1936) y Schubart (1929), donde su valor decrece de 3.0 a 1.2, cuando $\theta$ incrementa de 10 a 900, respectivamente, mientras que cuando $90<\theta \leq 180^{\circ}$, el valor $\delta_{1}$ se mantiene constante en 1.2. Sin embargo, el valor de $\delta_{2}$ se obtiene de tablas y gráficos con datos de Weisbach (1855), o bien mediante una ecuación propuesta por este autor, que al sustituirla en la ecuación anterior adopta la siguiente estructura: 
Tecnología y

Ciencias $₫$ Agua
2021, Instituto Mexicano de Tecnología del Agua

Open Access bajo la licencia CC BY-NC-SA 4.0

(https://creativecommons.org/licenses/by-nc-sa/4.0/)

$\xi_{S C}=\delta_{1}\left(0.95 \sin ^{2} \frac{\theta}{2}+2.05 \sin ^{4} \frac{\theta}{2}\right)$

g) Si la curva o codo son simples, con condición brusca $(r / D=0)$, de superficies rugosas $(\varepsilon>0)$ y $0<\theta \leq 180^{\circ}$, que conduce flujos con $R_{e} \geq$ 300, el coeficiente debe estimarse mediante la siguiente ecuación:

$\xi_{S C}=\delta_{1} \delta_{2} \eta_{1} \eta_{2}$

en la cual $\delta_{1}$ y $\delta_{2}$ se determinan conforme a lo indicado en $f$ ); $\eta_{1}$ y $\eta_{2}$ son coeficientes que se determinan en tablas en función del valor de $\varepsilon / D$ y $R_{e}$. El coeficiente $\eta_{1}$ puede incrementar de $45 f$ a 1.0 , cuando aumenta el valor de $R_{e}$, mientras que $\eta_{2}$ puede incrementar de 1.0 a 1.5 , cuando incrementan los valores de $\varepsilon / D$ y $R_{e}$.

h) Si se presentan dos curvas en serie de condición gradual, con superficies lisas $(\varepsilon \approx 0)$ y $r / D \geq 0.5$, formando una estructura en forma de $L, U \circ Z$, para conducir flujos con $R_{e} \geq 2 \times 10^{5}$, el coeficiente de pérdidas se determina mediante la siguiente ecuación:

$\xi_{G C}=K\left[\delta \alpha+\left(0.2 \frac{L_{s}-2 r}{D}+0.0007 \frac{r}{D} \theta\right)\right]$

donde los coeficientes $\delta$ y $\alpha$ se determinan conforme a lo indicado en a); $K$ es un coeficiente que depende de la relación $\left(L_{s}-2 r\right) / D$, el cual se estima mediante tablas obtenidas a partir de los resultados de Haase 
(1953). En el caso de las formas en $L$ y $Z$, el coeficiente $K$ disminuye de 2.5 a 2.0 y 3.0 a 2.0 , respectivamente, cuando la relación $\left(L_{s}-2 r\right) / D$ aumenta de 0 a $\geq 1.0$. Sin embargo, en el caso de la forma en $U$, dicho coeficiente incrementa de 1.4 a 2.0, cuando la relación $\left(L_{s}-2 r\right) / D$ aumenta de 0 a $\geq 1.0$.

i) Por último, cuando se presentan dos curvas graduales en serie, con superficies rugosas $(\varepsilon>0)$ y $r / D \geq 0.5$, estableciendo una estructura en forma-L, U o Z, para conducir flujos con $R_{e} \geq 0.03 \times 10^{5}$, el coeficiente de pérdidas se determina mediante la siguiente ecuación:

$\xi_{G C}=K\left[\delta \alpha \lambda_{1} \lambda_{2}+\left(0.2 \frac{L_{s}-2 r}{D}+0.0007 \frac{r}{D} \theta\right)\right]$

en la cual los coeficientes $\delta, \alpha, \lambda_{1}$ y $\lambda_{2}$ se obtienen conforme a lo indicado en la parte b) y c) para relaciones $0.5<r / D \leq 1.5$ y $1.5<r / D \leq 50$, respectivamente; y el coeficiente $K$ se estima de acuerdo con lo establecido en el apartado $\mathrm{h}$ ).

Por otro lado, King, Wislery Woodburn (1980) recomiendan valores tabulados para estimar el $\xi_{G C}$, $\operatorname{con} \theta=90^{\circ}$ y $1 \leq r / D \leq 20$, los cuales son obtenidos de los resultados de Beij (1938); dichos resultados se exponen de forma gráfica en Brater, King, Lindell y Wei (1996), y también se indica que en flujo turbulento el valor de $R_{e}$ no incide de modo significativo sobre el $\xi_{G C}$. En dichos datos, el valor de $\xi_{G C}$ disminuye de 0.35 a 0.16 , cuando $r / D$ aumenta de 1 a 4 , mientras que después incrementa de 0.16 a 0.42 , cuando $r / D$ toma valores de 4 a 20, respectivamente. Esto indica que el 
Teçnología y

Ciencias $₫$ Agua
2021, Instituto Mexicano de Tecnología del Agua

Open Access bajo la licencia CC BY-NC-SA 4.0

(https://creativecommons.org/licenses/by-nc-sa/4.0/)

valor mínimo de $\xi_{G C}=0.16$ es originado en $r / D=4$, mientras que el valor máximo de 0.42 es presentado en $r / D=20$. Por último, se establece de forma muy general que, en curvas graduales $\operatorname{con} \theta=45$ y $180^{\circ}$, el valor del $\xi_{G C}$ se puede determinar mediante el $50 \%$ y el $125 \%$, respectivamente, de los valores dados para la curva $\operatorname{con} \theta=90^{\circ}$.

El manual de USACE (1980) presenta lo establecido por Anderson (1947) para estimar el coeficiente de pérdidas en curvas graduales y bruscas, donde se enmarca lo siguiente:

a) Respecto a dispositivos graduales, se argumenta que Hofmann (1929) y Wasielewski (1932) establecieron que el $\xi_{G C}$ es independiente para $R_{e}>2$ $x 10^{5}$. Bajo este contexto, se recomienda un gráfico con resultados de Wasielewski (1932) para estimarel $\xi_{G C}$ con superficies lisas, $R_{e}=2.25 \mathrm{x}$ $10^{5}, 1 \leq r / D \leq 10$ y valores de $0<\theta \leq 90^{\circ}$; este gráfico también es sugerido por Sotelo (2013). Ahí, en forma general, el coeficiente incrementa a medida que aumenta el valor de $\theta$, pero disminuye conforme incrementa la relación $r / D$. Sin embargo, el coeficiente incrementa constantemente y toma el mismo valor en cualquier relación $r / D$ para los ángulos $\theta$ de 0 a $25^{\circ}$, mientras que si $r / D$ toma valores de 4,6 y 10 , el coeficiente decrece a partir de $\theta=65,70$ y $50^{\circ}$, respectivamente. También se ilustra que el valor máximo de $\xi_{G C}=0.20$ y se produce en $r / D$ $=1$ y $\theta=70^{\circ}$, mientras que el valor mínimo es cero para cualquier valor de $r / D$, cuando $\theta=0^{\circ}$.

b) Además, en el mismo gráfico se presentan curvas de ajuste sobre los resultados de Wasielewski (1932), las cuales recomienda USACE (1980) para obtener el $\xi_{G C}$ en función de $1.5 \leq r / D \leq 10$ y $0<\theta \leq 90^{\circ}$. Conforme 
Teçnología y

Ciencias $₫$ Agua
2021, Instituto Mexicano de Tecnología del Agua

Open Access bajo la licencia CC BY-NC-SA 4.0

(https://creativecommons.org/licenses/by-nc-sa/4.0/)

a este criterio, el $\xi_{G C}$ no presenta fluctuaciones y aumenta conforme incrementa el $\theta$, mientras que disminuye al aumentar $r / D$. También, los valores mínimos son cero cuando $\theta=0^{\circ}$, en tanto que el máximo es de 0.16 cuando $r / D=1.5$ y $\theta=90^{\circ}$.

c) Por último, si los dispositivos son bruscos, USACE (1980) y también Sotelo (2013) recomiendan un gráfico con resultados de Kirchbach (1929) y Schubart (1929) para estimar el $\xi_{S C}$, en función de los rangos $0.2 \leq R_{e} \leq$ $2.5 \times 10^{5}$ y $0 \leq \theta \leq 90^{\circ}$. En dicho gráfico el coeficiente incrementa a medida que aumenta $\theta$, mientras que disminuye cuando aumenta $R_{e}$. Los valores mínimos y máximos del $\xi_{S C}$ coinciden en $\theta=0^{\circ}$ y $90^{\circ}$, respectivamente, para cualquier valor de $r / D$; los valores mínimos son cero, mientras que los máximos resultan de 1.12 .

Otro documento que se cita en la literatura para determinar el coeficiente de pérdidas en curvas de tuberías es el manual de Crane Co. (1982), donde se recomiendan los valores de Pigott (1950) para obtener el $\xi_{G C}$ de tuberías de acero, $\operatorname{con} \theta=90^{\circ}, D$ aproximado de 2.0 a $25.4 \mathrm{~cm}$ y $r / D=1,2$ y 3 . Dichos datos revelan que el coeficiente disminuye en relación con el incremento del $D$, tomando valores aproximados de 3.8 a 1.8 , de 3.3 a 1.1 y de 3.5 a 1.25 , cuando $r / D$ vale 1,2 y 3 , respectivamente, lo cual indica que los valores máximos se obtienen con $r / D=1$, mientras que los mínimos con $r / D=2$. Además, también se exponen los resultados de Beij (1938) sobre el $\xi_{G C}$, los cuales fueron discutidos en USACE (1980). Sin embargo, si las curvas de $90^{\circ}$ son de acero y bridadas, o con extremos para soldar, se recomienda una tabla para estimar el $\xi_{G C}$, en función de la relación $r / D$ de 1 hasta 20 . En dicha tabla, el coeficiente decrece de $20 f$ a $12 f$ e incrementa hasta $50 f$, cuando 
$r / D$ toma valores de $1,2-3$ y 20 , respectivamente, presentándose los valores mínimos en $r / D$ de 2 a 3. También se especifica que para una curva con $\theta=180^{\circ}$ (forma-U, $L_{s}=0$ ), el $\xi_{G C}=50 \mathrm{f}$. Por último, se recomienda una tabla con resultados de Kirchbach (1929), para estimar el coeficiente en curvas bruscas de acero $\operatorname{con} \theta$ de 0 a $90^{\circ}$, donde el $\xi_{s c}$ incrementa de $2 f$ a $60 f$, respectivamente.

En SARH (1984) se sugiere una tabla para estimar el coeficiente de pérdidas de curvas graduales $\operatorname{con} \theta=90^{\circ}$, donde el $\xi_{G C}$ se rige por la relación $r / D$ en el rango de 1 a 10 . En dicha tabla, el $\xi_{G C}$ decrece de 0.52 a 0.18 , cuando $r / D$ aumenta de 1 a 6 , mientras que después incrementa de 0.18 a 0.20 conforme $r / D$ crece de 6 a 10. Sin embargo, si la curva es con $\theta \neq 90^{\circ}$, el valor del $\xi_{G C}$ obtenido será proporcional a un factor, el cual se estima de una segunda tabla en función de $\theta$; dicho factor incrementa de 0.20 a 1.30 acorde con el aumento de $\theta$ de 10 a $180^{\circ}$, respectivamente. Por último, se dice ordinariamente, si la curva es brusca, el $\xi_{S C}$ puede tomar valores de 0.7 a 1.0 , según la magnitud de $\theta$.

Por otro lado, Pashkov y Dolqachev (1985) exponen que en curvas graduales con $D \leq 3.0 \mathrm{~cm}$, el coeficiente de pérdidas puede obtenerse de una tabla sólo en función de $\theta$, donde el valor de $\xi_{G C}$ incrementa de 0.20 a 1.10, a medida que $\theta$ aumenta de 30 a $90^{\circ}$. Sin embargo, si la curva es brusca, el coeficiente se obtiene mediante la siguiente expresión:

$\xi_{S C}=\xi_{90}(1-\cos \theta)$ 
donde $\xi_{90}$ es el coeficiente de pérdidas de una curva brusca de $90^{\circ}$, que se estima de una tabla en función del $D$, en la cual el $\xi_{90}$ decrece de 1.70 a 0.83 , acorde con el incremento $D$ de 2.0 a $4.9 \mathrm{~cm}$, respectivamente.

En el diseño de presas pequeñas, USBR (1985) recomienda el gráfico discutido anteriormente en Beij (1938), para determinar el coeficiente de pérdidas de una curva gradual de $90^{\circ}$. No obstante, en dicho gráfico este autor incluye una curva de ajuste para obtener el $\xi_{G C}$ acorde con la relación $r / D$. Ahí el coeficiente disminuye de 0.25 a 0.07 cuando $r / D$ incrementa de 1 a 4 , respectivamente; luego se mantiene constante en 0.07 cuando $r / D$ va de 4 a 10 . Por otro lado, si la curva presenta un $\theta \neq 90^{\circ}$, se corrige el valor obtenido de $\xi_{G C}$, en proporción a un factor que se obtiene de un gráfico en función de $\theta$. Dicho factor incrementa de 0 hasta 1.12 , cuando $\theta$ aumenta de 0 a $120^{\circ}$, respectivamente.

Por otra parte, en Simon (1986) se sugiere una ecuación para estimar el coeficiente de pérdidas de curvas graduales, la cual se puede escribir de la siguiente forma:

$\xi_{G C}=\left[0.13+\frac{0.1635}{(r / D)^{3.5}}\right]\left(\frac{\theta}{180}\right)^{0.5}$

Además, si la curva se produce bruscamente, se indica que el coeficiente de pérdidas se obtiene utilizando la siguiente expresión:

$\xi_{S C}=67.6 \times 10^{-6}(\theta)^{2.17}$ 
La ecuación anterior también se recomienda para estimar el coeficiente de pérdidas en codos bruscos.

Por otro lado, de acuerdo con Trueba (1986), el coeficiente de pérdidas de una curva brusca se obtiene mediante la siguiente ecuación:

$\xi_{S C}=0.25\left(\frac{\theta}{90}\right)^{0.5}$

donde el ángulo $\theta$ debe ser de 0 a $90^{\circ}$.

Acorde con Miller (1990), en el caso de curvas graduales que presenten superficies lisas, relaciones $L_{d} / D \geq 30 \mathrm{y}$ valores de $R_{e}=10 \mathrm{x}$ $10^{5}$, el $\xi_{G C}$ se debe obtener de un gráfico en función de $0.5 \leq r / D \leq 10$ y $10 \leq \theta \leq 180^{\circ}$; dicho gráfico también es sugerido en CFE (1983) y Hager (2010). Ahí se exponen diversas curvas para obtener el $\xi_{G C}$, donde toma un mínimo valor de 0.02 y un máximo de 1.0. También se muestra que para cualquier valor del $\theta$ el coeficiente decrece a medida que incrementa la relación $r / D$, originándose los valores mínimos con $r / D$ entre 1.0 y 3.0; a partir de ahí, el valor del $\xi_{G C}$ incrementa conforme también aumenta $r / D$, pero no logra alcanzar valores como cuando $r / D<1.0$, donde se obtienen los valores máximos para cualquier valor de $\theta$. No obstante, al no cumplirse con paredes lisas o los valores mencionados para $L_{d} / D$ y $R_{e}$, en Miller (1990) y CFE (1983) se establece que el coeficiente de pérdidas obtenido del gráfico mencionado debe corregirse de la siguiente forma: 
Tecnología y

Ciencias $\stackrel{\Im}{\Im}$ Agua
2021, Instituto Mexicano de Tecnología del Agua

Open Access bajo la licencia CC BY-NC-SA 4.0

(https://creativecommons.org/licenses/by-nc-sa/4.0/)

$\xi_{G C}=\xi_{1} C_{R_{e}} C_{L_{d}} C_{\varepsilon}$

donde $\xi_{1}$ es el coeficiente de pérdidas obtenido del gráfico para tuberías lisas con relación $L_{d} / D \geq 30$ y en valores de $R_{e}=10 \times 10^{5} ; C_{R_{e}}, C_{L_{d}}$ y $C_{\varepsilon}$ son coeficientes de corrección por $R_{e} \neq 10 \times 10^{5}, L_{d} / D<30$ y rugosidad en las paredes del dispositivo, respectivamente. En el caso del coeficiente $C_{R_{e}}$, cuando $r / D \geq 1.0 \circ \xi_{1}<0.4$, este coeficiente se obtiene de un gráfico en función de $r / D \geq 1$ y $0.1 \leq R_{e} \leq 100 \times 10^{5}$, donde el valor de $C_{R_{e}}$ decrece a medida que incrementan los parámetros que lo rigen. Sin embargo, cuando $r / D<1.0$, el valor $C_{R_{e}}$ debe determinarse con la siguiente ecuación:

$C_{R_{e}}=\frac{\xi_{1}}{\xi_{1}-0.2 C_{R e 1}+0.2}$

donde $C_{R e 1}$ es el coeficiente de corrección por $R_{e} \neq 10 \times 10^{5}$ para $r / D \geq$ $1.0 \circ \xi_{1}<0.4$ (discutido previamente). Por otro lado, el coeficiente $C_{L_{d}}$ se determina de un gráfico en función del coeficiente $\xi_{1}$ y la relación $L_{d} / D$, donde sus valores máximos se presentan en $L_{d} / D=0$, mientras que sus valores mínimos se producen en $L_{d} / D$ de 1 a 4 . Por último, si $r / D \geq 1.0$ y $R_{e} \leq 10 \times 10^{5}$, el coeficiente $C_{\varepsilon}$ se determina como sigue:

$C_{\varepsilon}=\frac{f_{R}}{f_{L}}$ 
2021, Instituto Mexicano de Tecnología del Agua

Open Access bajo la licencia CC BY-NC-SA 4.0

(https://creativecommons.org/licenses/by-nc-sa/4.0/)

donde $f_{R}$ y $f_{L}$ son coeficientes de resistencia a la fricción en la tubería rugosa y en una tubería hidráulicamente lisa, respectivamente. Sin embargo, si $r / D \geq 1.0$ y $R_{e}>10 \times 10^{5}$, el $C_{\varepsilon}$ se obtiene con la ecuación anterior, pero obteniendo los coeficientes $f_{R}$ y $f_{L}$ con $R_{e}=10 \times 10^{5}$.

Finalmente, si la curva es brusca, Miller (1990) y CFE (1983) indican que el $\xi_{S C}$ se determine con el mismo proceder que para la curva gradual. Para ello, se recomienda un gráfico para estimar el $\xi_{S C}$ en función de $5 \leq$ $\theta \leq 120^{\circ}$, el cual aplica en tuberías lisas con $L_{d} / D \geq 30$ y $R_{e}=10 \times 10^{5}$; en caso contrario, el coeficiente debe corregirse de la misma forma a la condición gradual. En dicho gráfico, el $\xi_{s c}$ incrementa de 0.02 a 1.5, conforme aumenta el valor de $\theta$ de 5 a $120^{\circ}$, respectivamente.

Con base en Franzini y Finnemore (1999), se presenta un gráfico para estimar el coeficiente de pérdidas en curvas graduales $\operatorname{con} \theta=90^{\circ}$, donde el $\xi_{G C}$ se rige por $r / D$ y $\varepsilon / D$; cabe destacar que estos resultados no incluyen la pérdida por fricción en la curva. En este gráfico se muestra que el $\xi_{G C}$ incrementa a medida que aumenta la relación $\varepsilon / D$ para cualquier valor de $r / D$. No obstante, el coeficiente decrece e incrementa para cada valor de $\varepsilon / D$, cuando $r / D$ toma valores de 1 a 7 y de 7 a 10 , respectivamente, presentándose los valores mínimos en $r / D=7$ y los máximos en $r / D=1$. Por lo tanto, el valor mínimo es de 0.08 en $\varepsilon / D=0$ y el máximo de 0.90 en $\varepsilon / D=0.01$. Por último, se recomienda de forma general que para curvas $\operatorname{con} \theta=22.5$ y $45^{\circ}$, el $\xi_{G C}$ es aproximadamente el 40 y $80 \%$ del valor estimado para una curva $\operatorname{con} \theta=90^{\circ}$.

Mays (2001) recomienda una tabla para estimar el coeficiente de pérdidas en curvas graduales con superficies lisas y $\theta=45$ y $90^{\circ}$, en 
Tecnología y

Ciencias $₫$ Agua
2021, Instituto Mexicano de Tecnología del Agua

Open Access bajo la licencia CC BY-NC-SA 4.0

(https://creativecommons.org/licenses/by-nc-sa/4.0/)

función de $r / D$. En las curvas de $45^{\circ}$, el $\xi_{G C}$ decrece de 0.10 a 0.09 , cuando $r / D$ aumenta de 1 a 2, luego incrementa de 0.09 a 1.20 a medida que $r / D$ crece de 2 a 6 . Sin embargo, en las curvas de $90^{\circ}$ el $\xi_{G C}$ decrece 0.35 a 0.16 y luego incrementa de 0.16 a 0.21 , cuando $\xi_{G C}$ aumenta de 1 a 4 y de 4 a 6 , respectivamente. Por último, se indica que en curvas bruscas de $90^{\circ}$, el $\xi_{s c}$ puede tomar un valor de 1.10 , lo cual es bastante general.

Por su parte, Albers (2010) propone un gráfico para estimar el $\xi_{G C}$ si $\theta=90^{\circ}$, el cual también es aplicable para codos. Ahí el $\xi_{G C}$ decrece conforme incrementa $r / D$, alcanzando un valor máximo y mínimo de 1.0 y 0 , cuando $r / D$ se aproxima a 0 y 8 , respectivamente. Por otro lado, se indica que para una curva $\operatorname{con} \theta=45,135$ y $180^{\circ}$, el valor obtenido del $\xi_{G C}$ en el gráfico para $\theta=90^{\circ}$ será proporcional a $0.5,1.5$ y 2.0 , respectivamente.

Hager (2010), además de proponer el gráfico de Miller (1990) para $R_{e}=10 \times 10^{5}$, también sugiere otro gráfico para estimar el $\xi_{G C}$ con $R_{e} \geq$ $10 \times 10^{5}$, el cual también es sugerido en Ito (1960) y Blevins (1984). En este gráfico, el $\xi_{G C}$ puede obtenerse para $\theta=45,90$ y $180^{\circ}$, en función de $0.5 \leq r / D \leq 10$. Aunque dicho gráfico está representado de forma diferente, el comportamiento del $\xi_{G C}$ es similar al mencionado en Miller (1990). Los valores mínimos de $\xi_{G C}$ para $\theta=45$, 90 y $180^{\circ}$ son aproximados a $0.09,0.14$ y 0.18 , cuando $r / D=1.5,2.5$ y 2.0 , respectivamente; mientras que los valores máximos se aproximan a 0.25 , 0.6 y 0.6 , cuando $r / D=0.5,0.65$ y 0.75 , respectivamente. Por otro lado, si $R_{e}<10 \times 10^{5}$, el $\xi_{G C}$ obtenido en el gráfico mencionado debe multiplicarse por $\left(10 \times 10^{5} / R_{e}\right)^{1 / 6}$. 
2021, Instituto Mexicano de Tecnología del Agua

Open Access bajo la licencia CCBY-NC-SA 4.0

(https://creativecommons.org/licenses/by-nc-sa/4.0/)

A partir de Kast (2010), se recomienda un gráfico elaborado con resultados experimentales para estimar el $\xi_{G C}$ con superficies lisas o rugosas, $1 \leq r / D \leq 10$ y $15 \leq \theta \leq 180^{\circ}$, que conducen flujos con $R_{e} \geq 1$ $x$ 105. En dicho gráfico se muestra que el $\xi_{G C}$ para superficies lisas incrementa conforme también aumenta el $\theta$, mientras que respecto a $r / D$ presenta comportamientos distintos. Por ejemplo, cuando el ángulo $\theta=$ 15 y $30^{\circ}$, el $\xi_{G C}$ se mantiene constante cerca de 0.03 y 0.06 , respectivamente, para cualquier valor de $r / D$. Sin embargo, cuando $\theta=$ $45^{\circ}$ el $\xi_{G C}$ decrece a medida que incrementa $r / D$, tomando valores máximos y mínimos aproximados a 0.14 y 0.07 para $r / D=1$ y 10 , respectivamente. No obstante, si $\theta=90^{\circ}$, el $\xi_{G C}$ presenta un valor máximo aproximado a 0.22 en $r / D=1$, de donde decrece hasta un valor mínimo cercano a 0.09 en $r / D=6.5$, luego vuelve a incrementar hasta cerca de 0.11 cuando $r / D=10$. Ahora, si $\theta=180^{\circ}$, el coeficiente decrece de 0.32 a 0.13 , luego incrementa a 0.15 y decrece nuevamente hasta el valor mínimo de 0.11 , para finalmente incrementar hasta 0.15 ; dichos valores se presentan cuando $r / D$ vale $1,2.8,4,6.5$ y 10 , respectivamente. Por último, se muestra que para curvas rugosas con $\theta$ $=90^{\circ}$, el comportamiento del $\xi_{G C}$ es similar, pero con mayor magnitud al de las curvas lisas con el mismo $\theta$. Dicho coeficiente decrece desde el valor máximo de 0.50 hasta el valor mínimo de 0.18 , de donde incrementa hasta 0.20 ; esto ocurre cuando $r / D=1,6.5$ y 10 , respectivamente. Por otro lado, también se expone otro gráfico para obtener el $\xi_{G C}$ con superficies lisas, $\theta=90^{\circ}, 2.26 \leq r / D \leq 11.71$ y $20 \leq$ $R_{e} \leq 2 \times 10^{5}$. En éste se muestra que el $\xi_{G C}$ decrece a medida que incrementa el $R_{e}$ para cualquier relación $r / D$, presentando el valor máximo 
Tecnología y

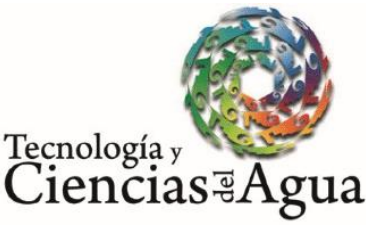

2021, Instituto Mexicano de Tecnología del Agua

Open Access bajo la licencia CC BY-NC-SA 4.0

(https://creativecommons.org/licenses/by-nc-sa/4.0/)

y mínimo cercano a 100 y 0.14 , cuando $R_{e}=20$ y 2 x $10^{5}$, respectivamente. Sin embargo, para $250 \leq R_{e} \leq 900$, el valor de $\xi_{G C}$ se mantiene constante cerca de 2.0. Finalmente, se sugiere información en tablas del coeficiente de pérdidas para curvas o codos estándar en condición brusca con $\theta=90^{\circ}$ tanto de conexiones atornilladas como elaborados de hierro fundido. Para los dispositivos de conexiones atornilladas con $r_{i}=0$ y $r_{o} \geq 0$, como en curvas con $r_{i} \geq 0$ y $r_{o}=0$, se recomienda una tabla para $D$ de 1.4 a $4.9 \mathrm{~cm}$, donde el $\xi_{S C}$ decrece de 1.2 a 0.51 , desde el $D$ mínimo al máximo, respectivamente. Ahora, si las curvas bruscas son de hierro fundido, se recomiendan los datos de Herning (1966) para $D$ de 5 a $50 \mathrm{~cm}$, donde el coeficiente incrementa de 1.3 a 2.2, conforme incrementa el $D$ mínimo hasta el máximo, respectivamente.

Menon y Menon (2010) presentan una tabla para estimar el coeficiente de pérdidas de curvas bruscas estándar, en función de $0<\theta$ $\leq 90^{\circ}$ y $D$ de 0.92 a $59.05 \mathrm{~cm}$ ( $D n$ de 0.5 a 24 pulgadas $\approx$ de 1.27 a 60.96 $\mathrm{cm})$, sin tomar en cuenta el valor de $R_{e}$. En dicha tabla se indica que el $\xi_{s c}$ incrementa conforme aumenta el valor de $\theta$, mientras que decrece cuando incrementa el valor del $D$. Por lo tanto, el valor mínimo del $\xi_{S C}=$ 0.02 , cuando $\theta \approx 0^{\circ}$ y $D=60.96 \mathrm{~cm}$, mientras que el máximo es de 1.62 , cuando $\theta=90^{\circ}$ y $D=1.27 \mathrm{~cm}$.

En el documento de Mataix (2010) se recomiendan valores tabulados del coeficiente de pérdidas, que son aplicables en curvas y codos graduales $\operatorname{con} \theta=90^{\circ}$. El valor de $\xi_{G C}$ es menor a medida que incrementa la relación $r / D$; el valor máximo del coeficiente es de 0.80 cuando $r / D \approx 0$, en tanto que el valor mínimo es de 0.16 con $r / D=1.0$. 
Respecto a Munson, Okiishi, Huebsch y Rothmayer (2013), se establece que el coeficiente de pérdidas de una curva o codo $\operatorname{con} \theta=90^{\circ}$, puede estimarse mediante un gráfico en función de $\varepsilon / D$ y $r / D$, con rangos de 0 a 0.01 y 1 a 10 , respectivamente. En este gráfico se revela que para cualquier valor de $\varepsilon / D$, el $\xi_{G C}$ decrece cuando $r / D$ va de 1 a 7 , donde alcanza los valores mínimos, luego incrementa hasta que $r / D=10 \sin$ superar los valores máximos que ocurren en $r / D=1$. Por lo tanto, en este caso el valor mínimo del $\xi_{G C} \approx 0.06$, cuando $\varepsilon / D \approx 0$ y $r / D=7$, mientras que el valor máximo es de 0.95 en $\varepsilon / D=0.01$ y $r / D=1$.

Acorde con Sotelo (2013), se establece que el coeficiente de pérdidas de una curva gradual con superficies rugosas, sea determinado mediante la siguiente ecuación:

$\xi_{G C}=C_{a} \frac{\theta}{90}$

donde $\theta$ puede ser de 0 a $90^{\circ} ; C_{a}$ es un coeficiente que se estima de un gráfico con resultados de Hofmann (1929) para flujos de $R_{e} \geq 2 \times 10^{5}$, donde está en función de las relaciones $r / D$ y $\varepsilon / D$, de 1 a 10 y de 0 a 0.002 , respectivamente. En este gráfico, el valor de $C_{a}$ crece a medida que incrementa la relación $\varepsilon / D$ para cualquiervalor de $r / D$; sin embargo, en todas las relaciones $\varepsilon / D$ dicho coeficiente disminuye y luego aumenta en el momento que $r / D$ incrementa de 1 a 7 y de 7 a 10 , respectivamente. Por lo tanto, el valor mínimo de $C_{a}$ se produce en $r / D=7$ y $\varepsilon / D \approx 0$, entretanto que el máximo en $r / D=1$ y $\varepsilon / D=0.002$. 
En Gülich (2014) se sugiere una ecuación para calcular el coeficiente de pérdidas en una curva brusca, la cual se muestra a continuación:

$\xi_{S C}=1.2\left(\frac{\theta}{90}\right)^{2}$

donde $\theta$ puede tomar valores de 0 a $90^{\circ}$. Por otro lado, también se sugiere una ecuación para estimarel coeficiente de pérdidas de curvas graduales, la cual se escribe de la siguiente manera:

$\xi_{G C}=C_{\varepsilon} \frac{0.23}{(r / D)^{x}} \sqrt{\frac{\theta}{90}}$

en la cual $C_{\varepsilon}$ es un coeficiente de rugosidad que en superficies lisas es igual a la unidad, mientras que en superficies rugosas con $\varepsilon / D>0.001 \mathrm{y}$ $R_{e}>0.4 \times 10^{5}$, dicho coeficiente es igual a $2 ; x$ es un exponente cuyo valor es de 2.5 y 0.5 , cuando $0.5 \leq r / D \leq 1.0$ y $r / D>1.0$, respectivamente. 
Teçnología y

Ciencias $\stackrel{\unlhd}{\longleftarrow}$ Agua
2021, Instituto Mexicano de Tecnología del Agua

Open Access bajo la licencia CC BY-NC-SA 4.0

(https://creativecommons.org/licenses/by-nc-sa/4.0/)

Sumario de las metodologías para $\xi_{C}$

En la Tabla 1 se expone un sumario de las metodologías tratadas en el apartado anterior para estimar el coeficiente de pérdidas en curvas simples e instaladas en serie con forma-Z, forma-U, forma-L y forma-S, donde se indican características importantes y los parámetros considerados en cada una de ellas.

Tabla 1. Características y parámetros considerados para estimar el coeficiente de pérdidas en las metodologías analizadas.

\begin{tabular}{|c|c|c|c|c|c|c|c|c|}
\hline $\begin{array}{c}\text { Ref. } \\
\text { (fuente) }\end{array}$ & $\begin{array}{l}\text { Fluido } \\
\text { usado }\end{array}$ & Material & $\begin{array}{c}D \\
(\mathrm{~cm})\end{array}$ & $r / D$ & $\begin{array}{l}\theta^{\circ} 0 \\
\text { forma }\end{array}$ & $\begin{array}{c}R_{e} \\
\left(\times 10^{5}\right)\end{array}$ & $\begin{array}{l}L_{u} / L_{d} \\
(x D)\end{array}$ & Observaciones \\
\hline $\begin{array}{c}\text { Brightmore } \\
\text { (1907) }\end{array}$ & Agua & Acero & $\begin{array}{c}7.62 \\
10.16\end{array}$ & 2.5 a 10 & 90 & I & NS & Gráficos de $\xi_{G C}$ \\
\hline $\begin{array}{l}\text { Davis } \\
\text { (1911) }\end{array}$ & Agua & Acero & 5.08 & $\begin{array}{l}5.0 \mathrm{a} \\
20.5\end{array}$ & 90 & I & NS & Gráficos de $\xi_{G C}$ \\
\hline $\begin{array}{c}\text { Balch } \\
\text { (1913) }\end{array}$ & Agua & Acero & 7.62 & 7.0 a 20 & 90 & I & NS & Gráficos de $\xi_{G C}$ \\
\hline
\end{tabular}


Tecnología y

Ciencias $\approx$ Agua
2021, Instituto Mexicano de Tecnología del Agua

Open Access bajo la licencia CCBY-NC-SA 4.0

(https://creativecommons.org/licenses/by-nc-sa/4.0/)

\begin{tabular}{|c|c|c|c|c|c|c|c|c|}
\hline $\begin{array}{l}\text { Hofmann } \\
\text { (1929) }\end{array}$ & Agua & Acero & 4.32 & 1.0 a 10 & 90 & I & NS & $\begin{array}{l}\text { Gráficos de } \xi_{G C,} \\
\text { para superficies } \\
\text { lisas y rugosas }\end{array}$ \\
\hline $\begin{array}{c}\text { Gibson } \\
(1930) \\
\text { Weisbach } \\
\text { (1855) } \\
\text { Brightmore } \\
\text { (1907) } \\
\text { Schoder } \\
(1908)\end{array}$ & Agua & $\begin{array}{l}\text { Acero, } \\
\text { hierro } \\
\text { forjado y } \\
\text { fundido }\end{array}$ & $\begin{array}{c}3.17 \\
7.62 y \\
10.16 \\
15.40\end{array}$ & $\begin{array}{c}- \\
2.0 \text { a } 10 \\
1.34 \mathrm{a} \\
20\end{array}$ & 90 & I & NS & $\begin{array}{c}\text { Ecuaciones (21- } \\
22) \text { y tablas } \\
\text { para } \xi_{S c} \text { y } \xi_{G C}\end{array}$ \\
\hline $\begin{array}{c}\text { Vogel } \\
\text { (1933) }\end{array}$ & Agua & Acero & $\begin{array}{l}15.24 \\
20.32 \\
25.40\end{array}$ & 1.0 a 3.0 & 90 & I & NS & Gráficos de $\xi_{G C}$ \\
\hline $\begin{array}{c}\text { Beij } \\
(1938)\end{array}$ & Agua & Acero & 10.23 & $\begin{array}{l}0.97 a \\
19.96\end{array}$ & 90 & $\begin{array}{c}0.23 \mathrm{a} \\
3.4\end{array}$ & $\begin{array}{l}48.15 / \\
168.12\end{array}$ & $\begin{array}{c}\text { Tablas y } \\
\text { gráficos de } \xi_{G C}, \\
D n=4 \text { plg, } \\
\text { Resultados } \\
\text { comparados } \\
\text { con: Brightmore } \\
(1907) \\
\text { Davis } \\
(1911) \\
\text { Balch }\end{array}$ \\
\hline
\end{tabular}


2021, Instituto Mexicano de Tecnología del Agua

Tecnologiay

Open Access bajo la licencia CC BY-NC-SA 4.0

(https://creativecommons.org/licenses/by-nc-sa/4.0/)

\begin{tabular}{|c|c|c|c|c|c|c|c|c|}
\hline & & & & & & & & $\begin{array}{c}(1913) \\
\text { Hofmann } \\
(1929) \\
\text { Vogel } \\
(1933)\end{array}$ \\
\hline $\begin{array}{c}\text { Daugherty e } \\
\text { Ingersoll } \\
(1954) \\
\text { Pigott } \\
(1950)\end{array}$ & Agua & NS & NS & AV & $\begin{array}{c}0 a \\
180\end{array}$ & If & NS & $\begin{array}{l}\text { Ecuación ( } 23 \text { ) } \\
\text { para curvas } \\
\text { graduales que } \\
\text { depende de } f \text {. }\end{array}$ \\
\hline $\begin{array}{c}\text { Ito } \\
(1956)\end{array}$ & Agua & $\begin{array}{l}\text { Latón } \\
\text { fundido }\end{array}$ & 3.5 & $\begin{array}{c}28.4 \text { y } \\
108 \\
7.6 a \\
29.2 \\
5.8 \text { y } \\
9.05 \\
5.2 \text { y } 7.4\end{array}$ & $\begin{array}{r}45 \\
90 \\
135 \\
U\end{array}$ & $\begin{array}{c}0.1 \mathrm{a} \\
3.0\end{array}$ & NS & $\begin{array}{c}\text { Gráficos de } \xi_{G C} \\
\text { para curvas de } \\
\text { radio largo y } \\
\text { superficies lisas }\end{array}$ \\
\hline $\begin{array}{c}\text { Ito } \\
(1960)\end{array}$ & Agua & $\begin{array}{l}\text { Latón } \\
\text { fundido }\end{array}$ & 3.5 & $\begin{array}{c}1.84 \mathrm{a} \\
7.3 \\
1.0 \mathrm{a} 3.3 \\
1.84 \mathrm{y} \\
3.26\end{array}$ & $\begin{array}{c}45 \\
90 \\
U\end{array}$ & $\begin{array}{c}0.2 \mathrm{a} \\
4.0\end{array}$ & $\begin{array}{l}153.8 / \\
>71.6\end{array}$ & $\begin{array}{c}\text { Gráficos y } \\
\text { ecuaciones (7- } \\
\text { 12) de } \xi_{G C} \text { para } \\
\text { superficies } \\
\text { lisas. } \\
\text { Resultados } \\
\text { comparados }\end{array}$ \\
\hline
\end{tabular}


Ciencias $\approx$ A

2021, Instituto Mexicano de Tecnología del Agua

Open Access bajo la licencia CC BY-NC-SA 4.0

(https://creativecommons.org/licenses/by-nc-sa/4.0/)

\begin{tabular}{|c|c|c|c|c|c|c|c|c|}
\hline & & & & & & & & $\begin{array}{c}\text { con: Weisbach } \\
\qquad(1855) \\
\text { Hofmann } \\
(1929) \\
\text { Richter (1930) } \\
\text { Wasielewski } \\
\text { (1932) } \\
\text { Pigott (1950) } \\
\text { Pigott (1957) }\end{array}$ \\
\hline $\begin{array}{c}\text { Idel'chik } \\
(1966) \\
\text { Abramovich, } \\
(1935) \\
\text { Nekrasov } \\
(1954) \\
\text { Idel'chik } \\
(1953) \\
\text { Evdomikov } \\
(1940)\end{array}$ & NS & NS & NS & $\begin{array}{l}\geq 0.5 \\
0.5 a \\
1.5^{*} \\
1.5 a \\
50^{*}\end{array}$ & $\begin{array}{c}0 \mathrm{a} \\
180(\mathrm{U})\end{array}$ & $\begin{array}{c}\geq 2.0 \\
\geq 0.03 * \\
\geq 0.03 *\end{array}$ & NS & $\begin{array}{l}\text { Tablas, gráficos } \\
\text { y ecuaciones } \\
(24-30) \text { de } \xi_{c} \text {, } \\
\text { para superficies } \\
\text { lisas y rugosas* }\end{array}$ \\
\hline $\begin{array}{c}\text { Idel'chik } \\
(1966) \\
\text { Aronov } \\
(1950) \\
\text { Adler } \\
(1934)\end{array}$ & NS & NS & NS & $\gg 1.5$ & 0 a 90 & $\begin{array}{c}0.0005 \\
\text { a } 0.2\end{array}$ & NS & $\begin{array}{c}\text { Tablas, gráficos } \\
\text { y ecuaciones } \\
(31-34) \text { de } \xi_{c} \\
\text { en superficies } \\
\text { lisas }\end{array}$ \\
\hline
\end{tabular}


2021, Instituto Mexicano de Tecnología del Agua

Open Access bajo la licencia CCBY-NC-SA 4.0

(https://creativecommons.org/licenses/by-nc-sa/4.0/)

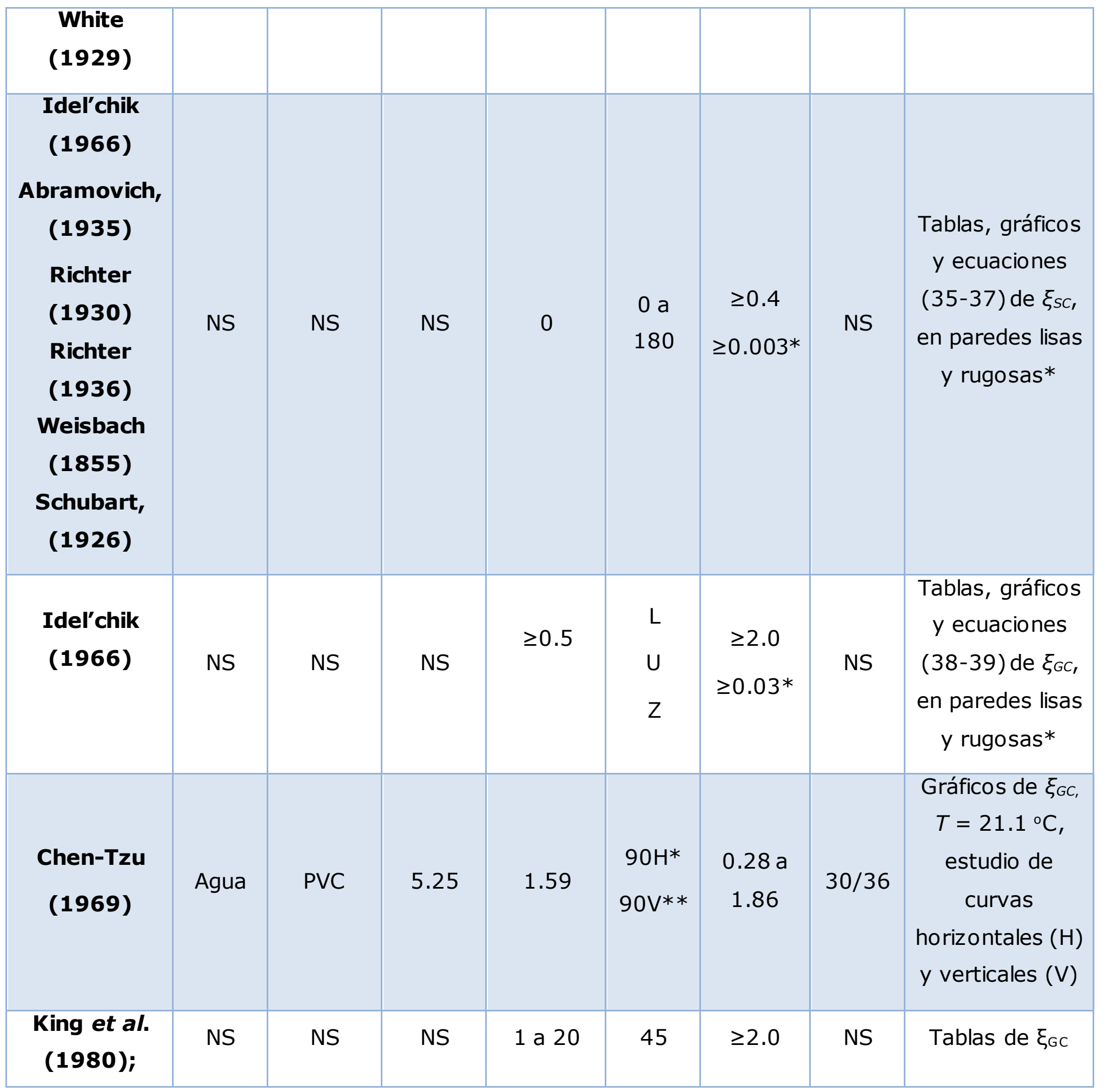


2021, Instituto Mexicano de Tecnología del Agua

Ciencias Ciengua

Open Access bajo la licencia CC BY-NC-SA 4.0

(https://creativecommons.org/licenses/by-nc-sa/4.0/)

\begin{tabular}{|c|c|c|c|c|c|c|c|c|}
\hline $\begin{array}{c}\text { Brater et al. } \\
(1996) \\
\text { Beij (1938) }\end{array}$ & & & & & $\begin{array}{c}90 \\
180\end{array}$ & & & \\
\hline $\begin{array}{c}\text { USACE } \\
\text { (1980); } \\
\text { Sotelo } \\
(2013) \\
\text { Anderson, } \\
\text { (1947) } \\
\text { Hofmann } \\
\text { (1929) } \\
\text { Wasielewski } \\
\text { (1932) } \\
\text { Kirchbach } \\
\text { (1929) } \\
\text { Schubart } \\
\text { (1929) }\end{array}$ & Agua & NS & NS & $\begin{array}{c}1 \text { a } 10 \\
\text { 0* }\end{array}$ & 0 a 90 & $\begin{array}{l}\geq 2.0 \\
0.2 \mathrm{a} \\
2.25^{*}\end{array}$ & NS & $\begin{array}{c}\text { Gráficos de } \xi_{G C} \\
\text { y } \xi_{s C^{*}}\end{array}$ \\
\hline $\begin{array}{c}\text { Crane Co. } \\
\text { (1982) } \\
\text { Pigott } \\
(1950) \text { Beij } \\
(1938) \\
\text { Kirchbach, } \\
(1929)\end{array}$ & Agua & Acero & $\begin{array}{c}1.27 \text { to } \\
60.96\end{array}$ & 1.0 a 20 & $\begin{array}{c}90 \\
180 \\
0 \mathrm{a} \\
90 *\end{array}$ & I & NS & $\begin{array}{c}\text { Tablas y } \\
\text { gráficos de } \xi_{S c} * \\
\text { y } \xi_{G C} \\
\text { Dispositivos de } \\
\text { acero y con } \\
\text { extremos } \\
\text { bridados }\end{array}$ \\
\hline $\begin{array}{c}\text { Turian et al. } \\
\text { (1983) }\end{array}$ & $\begin{array}{l}\text { Agua- } \\
\text { Solidos }\end{array}$ & Acero & $\begin{array}{l}2.54 \\
5.08\end{array}$ & $\begin{array}{l}1.0 \mathrm{a} \\
8.33\end{array}$ & $\begin{array}{c}90-S \\
45\end{array}$ & $\begin{array}{c}0.1 \mathrm{a} \\
0.8\end{array}$ & NS & $\begin{array}{c}\text { Tablas y } \\
\text { gráficos de } \xi_{s c} y\end{array}$ \\
\hline
\end{tabular}


2021, Instituto Mexicano de Tecnología del Agua

Ciencias

Open Access bajo la licencia CC BY-NC-SA 4.0

(https://creativecommons.org/licenses/by-nc-sa/4.0/)

\begin{tabular}{|c|c|c|c|c|c|c|c|c|}
\hline Hsu (1981) & & & & $\begin{array}{l}1.0 \mathrm{a} \\
11.1\end{array}$ & $\begin{array}{c}90-G \\
U\end{array}$ & $\begin{array}{c}0.2 \mathrm{a} \\
1.7\end{array}$ & & $\begin{array}{c}\xi_{G C}, \text { Flujo de } \\
\text { agua con perlas } \\
\text { de vidrio. } \\
\text { Resultados } \\
\text { comparados } \\
\text { con Crane Co. } \\
(1982)\end{array}$ \\
\hline $\begin{array}{l}\text { Gontsov et } \\
\text { al. (1984) }\end{array}$ & Aire & $\begin{array}{c}\text { Vidrio } \\
\text { Orgánico }\end{array}$ & 20.6 & 1.0 & 90 & $\begin{array}{c}0.9 \mathrm{a} \\
4.0\end{array}$ & $35 / 25$ & $\begin{array}{c}\text { Tablas y } \\
\text { ecuaciones (13- } \\
\text { 15) de } \xi_{G C} \text { para } \\
\text { superficies } \\
\text { lisas. } \\
\text { Resultados } \\
\text { comparados } \\
\text { con Ito (1960) }\end{array}$ \\
\hline $\begin{array}{l}\text { SARH } \\
(1984)\end{array}$ & Agua & NS & NS & 1.0 a 10 & $\begin{array}{c}0 \mathrm{a} \\
180\end{array}$ & I & NS & Tablas de $\xi_{G c}$ \\
\hline $\begin{array}{c}\text { Pashkov y } \\
\text { Dolqachev } \\
\text { (1985) }\end{array}$ & NS & NS & $\leq 3.0$ & I & $\begin{array}{c}0 \mathrm{a} \\
180\end{array}$ & I & NS & $\begin{array}{c}\text { Tablas de } \xi_{G C} Y \\
\text { ecuación }(40) \\
\text { de } \xi_{s c^{*}}\end{array}$ \\
\hline $\begin{array}{c}\text { USBR } \\
(1985) \\
\text { Beij (1938) }\end{array}$ & Agua & Acero & NS & 1 a 10 & $\begin{array}{c}0 \mathrm{a} \\
120\end{array}$ & I & NS & Gráficos de $\xi_{G C}$ \\
\hline $\begin{array}{l}\text { Simon } \\
(1986)\end{array}$ & NS & NS & NS & $\begin{array}{l}\text { AV } \\
0 *\end{array}$ & $\begin{array}{c}0 \mathrm{a} \\
180\end{array}$ & I & NS & $\begin{array}{c}\text { Ecuaciones (41- } \\
42) \text { de } \xi_{s c} * y \\
\xi_{G C .}\end{array}$ \\
\hline
\end{tabular}


2021, Instituto Mexicano de Tecnología del Agua

Tecnologiay

Open Access bajo la licencia CC BY-NC-SA 4.0

(https://creativecommons.org/licenses/by-nc-sa/4.0/)

\begin{tabular}{|c|c|c|c|c|c|c|c|c|}
\hline $\begin{array}{l}\text { Trueba } \\
\text { (1986) }\end{array}$ & NS & NS & NS & 0 & 0 a 90 & I & NS & $\begin{array}{c}\text { Ecuación (43) } \\
\text { de } \xi_{s c}\end{array}$ \\
\hline $\begin{array}{c}\text { Miller } \\
(1990) ; \\
\text { CFE (1983) }\end{array}$ & NS & NS & NS & $\begin{array}{c}0.5 \text { a } 10 \\
0 *\end{array}$ & $\begin{array}{l}10 a \\
180 \\
5 a \\
20 *\end{array}$ & $\begin{array}{c}0.1 \mathrm{a} \\
100\end{array}$ & $\begin{array}{l}\text { NS / } \\
\geq 0\end{array}$ & $\begin{array}{c}\text { Gráficos y } \\
\text { ecuaciones (44- } \\
46 \text { ) de } \xi_{G C} \text { y } \\
\xi_{S C} * \text { para } \\
\text { superficies lisas } \\
\text { y rugosas. Se } \\
\text { consideran } \\
\text { correcciones } \\
\text { por } R e, L_{d} \text { y } \varepsilon\end{array}$ \\
\hline $\begin{array}{c}\text { Franzini \& } \\
\text { Finnemore } \\
\text { (1999) }\end{array}$ & NS & NS & NS & 1.0 a 10 & $\begin{array}{c}22.5 \\
45 y \\
90\end{array}$ & I & NS & $\begin{array}{l}\text { Gráfico de } \xi_{G C,} \\
\text { donde también } \\
\text { se rige por } \varepsilon / D\end{array}$ \\
\hline $\begin{array}{l}\text { Mays } \\
(2001)\end{array}$ & NS & NS & NS & 1.0 a 6.0 & $\begin{array}{c}45 y \\
90 \\
90 *\end{array}$ & I & NS & $\begin{array}{c}\text { Tabla de } \xi_{G C} y \\
\xi_{s c} * \text { para } \\
\text { superficies lisas }\end{array}$ \\
\hline $\begin{array}{l}\text { Albers } \\
(2010)\end{array}$ & NS & NS & NS & 0 a 8.0 & $\begin{array}{c}45 \\
90 \\
135 y \\
180\end{array}$ & I & NS & $\begin{array}{l}\text { Gráfico de } \xi_{G c .} \\
\text { También es } \\
\text { aplicable para } \\
\text { codos. }\end{array}$ \\
\hline $\begin{array}{c}\text { Hager } \\
(2010) \\
\text { Miller } \\
(1990) \\
\text { Ito }(1960)\end{array}$ & NS & NS & NS & 0.5 a 10 & $\begin{array}{l}45 a \\
180\end{array}$ & $\begin{array}{l}\geq 10 \\
<10\end{array}$ & NS & Gráficos de $\xi_{G C}$ \\
\hline
\end{tabular}


2021, Instituto Mexicano de Tecnología del Agua

Ciencias

Open Access bajo la licencia CC BY-NC-SA 4.0

(https://creativecommons.org/licenses/by-nc-sa/4.0/)

\begin{tabular}{|c|c|c|c|c|c|c|c|c|}
\hline $\begin{array}{l}\text { Blevins } \\
(1984)\end{array}$ & & & & & & & & \\
\hline $\begin{array}{c}\text { Kast } \\
(2010)\end{array}$ & NS & Hierro & $\begin{array}{l}1.4 \text { to } \\
50 *\end{array}$ & $\begin{array}{c}1.0 \text { a } 10 \\
0 *\end{array}$ & $\begin{array}{l}15 a \\
180 \\
0 a \\
90 *\end{array}$ & $\geq 0.04$ & NS & $\begin{array}{c}\text { Tablas y } \\
\text { gráficos de } \xi_{s C^{*}} \\
\text { y } \xi_{G C} \text { para } \\
\text { superficies lisas } \\
\text { y rugosas. } \\
\text { También se } \\
\text { exponen tablas } \\
\text { de } \xi_{s C^{*}} \text { para } r_{i} \\
\geq 0 \text { y } r_{0} \geq 0\end{array}$ \\
\hline $\begin{array}{c}\text { Menon y } \\
\text { Menon } \\
(\mathbf{2 0 1 0 )}\end{array}$ & NS & NS & $\begin{array}{l}0.92 \mathrm{a} \\
59.05\end{array}$ & 0 & 0 a 90 & I & NS & $\begin{array}{l}\text { Tablas de } \xi_{s c} \text { en } \\
\text { curvas estándar }\end{array}$ \\
\hline $\begin{array}{l}\text { Mataix } \\
(2010)\end{array}$ & NS & NS & NS & 0 a 1.0 & 90 & I & NS & $\begin{array}{l}\text { Tablas de } \xi_{s c} \\
\text { que también } \\
\text { son aplicables } \\
\text { en codos }\end{array}$ \\
\hline $\begin{array}{c}\text { Munson et } \\
\text { al. } \\
\text { (2013) }\end{array}$ & NS & NS & NS & 1.0 a 10 & 90 & I & NS & $\begin{array}{c}\text { Tablas de } \xi_{s c} \\
\text { que también } \\
\text { son aplicables } \\
\text { en codos, } \\
\text { donde también } \\
\text { se rige por } \varepsilon / D\end{array}$ \\
\hline $\begin{array}{l}\text { Sotelo } \\
(2013)\end{array}$ & NS & NS & NS & 1.0 a 10 & 0 a 90 & $\geq 2.0$ & NS & $\begin{array}{c}\text { Gráfico y } \\
\text { ecuación (47) } \\
\text { de } \xi \text { sc para }\end{array}$ \\
\hline
\end{tabular}


Tecnología y

Ciencias $₫$ Agua
2021, Instituto Mexicano de Tecnología del Agua

Open Access bajo la licencia CC BY-NC-SA 4.0

(https://creativecommons.org/licenses/by-nc-sa/4.0/)

\begin{tabular}{|c|c|c|c|c|c|c|c|c|}
\hline $\begin{array}{c}\text { Hofmann, } \\
(1929)\end{array}$ & & & & & & & & $\begin{array}{c}\text { superficies } \\
\text { rugosas, donde } \\
\text { también se rige } \\
\text { por } \varepsilon / D\end{array}$ \\
\hline $\begin{array}{c}\text { Gülich } \\
\text { (2014) }\end{array}$ & NS & NS & NS & $\begin{array}{c}0 * \\
\geq 0.5\end{array}$ & 0 a 90 & I & NS & 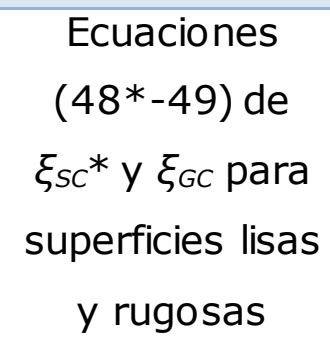 \\
\hline $\begin{array}{l}\text { Villegas- } \\
\text { León et al. } \\
\text { (2016) }\end{array}$ & NS & NS & NS & $\begin{array}{c}0 * \\
1.0 \text { a } 10\end{array}$ & $\begin{array}{c}0 \text { a } \\
90 * \\
5 \text { a } 90\end{array}$ & I & NS & $\begin{array}{c}\text { Ecuaciones (16- } \\
20) \text { de } \xi_{s c^{*} y} \\
\xi_{G C}\end{array}$ \\
\hline
\end{tabular}

NS = no especificado; I = independiente de su valor; $\mathrm{AV}=$ cualquier valor es válido; If = implícito en el valor del coeficiente $f$.

La Tabla 1 permite contrastar los fluidos y tipos de materiales de las curvas utilizadas en las investigaciones del coeficiente de pérdidas en curvas, los valores considerados de $D, r / D, \theta, R_{e}, L_{u} y L_{d}$, así como observaciones de los resultados presentados. En dichos datos se indica que el fluido más utilizado en las pruebas es el agua y que el tipo de material más analizado es el acero y el hierro. También se puede observar que las investigaciones se han realizado hasta $D \approx 60 \mathrm{~cm}$ (Crane Co., 1982; Menon \& Menon, 2010), pero la mayoría han sido en $D$ pequeños cercanos de $10 \mathrm{~cm}$ (4 pulgadas). La relación $r / D$ más grande investigada es de 108 cm (Ito, 1956), sin embargo, la más analizada es en el rango $r / D \leq 10$. Respecto al ángulo $\theta$ y tipo de forma se percata que las curvas 
2021, Instituto Mexicano de Tecnología del Agua

Open Access bajo la licencia CC BY-NC-SA 4.0

(https://creativecommons.org/licenses/by-nc-sa/4.0/)

más estudiadas son de condición gradual $\operatorname{con} \theta=90^{\circ}$ y las estructuras en forma-U con $L_{s}=0$; a partir de los valores obtenidos del coeficiente de pérdidas $\operatorname{con} \theta=90^{\circ}$, algunos autores como Franzini y Finnemore (1999) y Albers (2010) recomiendan inferir valores para ángulos distintos, lo cual puede conllevar a grandes errores en la estimación de las pérdidas de energía. Por otro lado, se ilustra que metodologías consideran el valor de $R_{e}$, su rango de validez y cuáles lo consideran independiente para obtener el coeficiente de las curvas; factor que es importante para estimar un coeficiente más apegado a la realidad, ya que en documentos como Turian et al. (1983), Kast (2010) y Hager (2010) se puede percatar que afecta su valor en forma decreciente a medida que incrementa $R_{e}$. Otro parámetro importante que se confronta es hasta que $L_{d}$ se analizaron las pérdidas de energía, pues se ha demostrado que hasta aproximadamente $L_{d}=50 D$ (Ito, 1956; Ito, 1960) aún incide en la pérdida de energía originada por las curvas de tuberías. En la última columna se presentan observaciones importantes, donde se indica qué coeficiente se determina $\left(\xi_{S C}, \xi_{G C}\right)$; la forma de presentación de resultados (gráficos, tablas o ecuaciones), así como para qué tipo se superficies son válidos (lisas o rugosas). Finalmente, cabe destacar que varias metodologías no hacen referencia del tipo de material y diámetro utilizados en los estudios, lo cual implica usarlos de forma general, pudiendo diferir sustancialmente en las pérdidas reales. 


\section{Conclusiones}

- Se realizó una revisión de literatura sobre investigaciones experimentales y metodologías reportadas, que permite estimarel coeficiente de pérdidas en curvas de tuberías bajo flujo turbulento con condiciones buscas y graduales, las cuales pueden presentarse de forma simple e instaladas en serie, para conformar estructuras con formas tipo L, S, U y Z. A partir de ello, se presentó información organizada y homogenizada que facilita comparar los alcances de las metodologías, así como el comportamiento del coeficiente de pérdidas ante los parámetros que lo rigen.

- Mediante las investigaciones y metodologías anal izadas de la literatura se obtienen valores distintos del coeficiente de pérdidas en los dispositivos estudiados, lo cual puede deberse a diferencias adoptadas en los experimentos, tales como el material de fabricación del dispositivo, la altura de rugosidad de las paredes interiores, el diámetro, el radio de curvatura, los ángulos de deflexión, la temperatura y velocidades del flujo, el tipo de fluido utilizado, tipos de conexión, la precisión de los dispositivos de medición y las longitudes de las tuberías aguas arriba y aguas abajo de los dispositivos. Sin embargo, todas estas características permiten una elección más acorde con un caso particular de la 
2021, Instituto Mexicano de Tecnología del Agua

Open Access bajo la licencia CC BY-NC-SA 4.0

(https://creativecommons.org/licenses/by-nc-sa/4.0/)

metodología o los valores a utilizar en la estimación del coeficiente de pérdidas.

- Los coeficientes de pérdidas reportados en las curvas de tuberías pueden incluir o no la pérdida por fricción sobre la longitud de la curva $\left(h_{f}\right)$, repercutiendo en el valor del coeficiente $y$, aún más, cuando los dispositivos son de radio de curvatura largo. Además de ello, también influye en el coeficiente la distancia considerada aguas abajo del dispositivo para la evaluación de la pérdida por perturbación del flujo $\left(h_{d}\right)$, la cual se ha demostrado que puede llegar hasta $50 \mathrm{D}$.

- En las investigaciones se manifestó que el coeficiente de pérdidas tiene dependencia sobre el ángulo $\theta$, la relación $r / D$, el valor de $R_{e}$ y la relación $\varepsilon / D$. No obstante, a menudo en las metodologías de la literatura, el coeficiente solo se expone en función de $\theta$ y $r / D$, pero el parámetro más excluido es la rugosidad de la superficie o la relación $\varepsilon / D$. En general, el coeficiente de pérdidas presentó incremento conforme al aumento de $\theta$ y $\varepsilon / D$, pero una disminución en relación con el crecimiento de $R_{e}$. En los dispositivos de condición gradual, el coeficiente aumentó a medida que decreció la relación $r / D$, mientras que en las cuatro formas con curvas en serie el coeficiente creció conforme al incremento de la longitud de separación entre los dispositivos $\left(L_{s}\right)$.

- Los dispositivos más estudiados y de los cuales se presenta más información del coeficiente de pérdidas son las curvas con $\theta=45,90$ y $180^{\circ}$. Sin embargo, el más investigado de ellos por su frecuente uso es el dispositivo con $\theta=90^{\circ}$, sobre el cual algunos autores infieren el coeficiente de pérdidas para curvas de 45 y $180^{\circ}$ de deflexión. Referente 
al material del dispositivo, los cambios de dirección con más investigaciones son los de acero, mientras los menos analizados son los modernos (plástico, moldeados y forjados), que tienden a proporcionar menores valores del coeficiente de pérdidas por su baja rugosidad.

- Finalmente, se concluye que la información de los trabajos de Ito (1959, 1960) es la más sugerida en la literatura para estimar el coeficiente de pérdidas en los dispositivos graduales, en tanto que en los dispositivos bruscos se recomiendan con más frecuencia los datos del trabajo de Kirchbach (1929). Sin embargo, donde se proporciona amplia información sobre el coeficiente de pérdidas de todos los dispositivos estudiados con respecto a sus parámetros dependientes es en el manual de resistencia de Idel'chik (1966).

\section{Referencias}

Abramovich, G. N. (1935). Aerodinamika mestnykh soprotivlenii (Aerodynamics of local resistances). Sbornik Po Promyshlennoi Aerodinamike, Trudy, 211, 65-150.

Acero, M. F., \& Rodríguez, D. (May, 2008). Determinacion experimental del coeficiente de pérdidas menores $\mathrm{km}$ en accesorios para agua potable de PVC. XVIII Seminario Nacional de Hidráulica e Hidrología, Sociedad Colombiana de Ingenieros. Recuperado de https://es.scribd.com/document/48818587/02-DeterminacionExperimental-de-km-Accesorios-PVC-P

Adler, M. (1934). Strömung in gekrümmten Röhren. Zeitschrift für Angewandte Mathematik und Mechanik, 14, 257-275. 
Albers, P. (2010). Motion control in offshore and dredging. Recuperado de https://doi.org/10.1007/978-90-481-8803-1

Anaya-Durand, A. I., Cauich-Segovia, G. I., Funabazama-Bárcenas, O., \& Gracia-Medrano-Bravo, V. A. (2014). Evaluación de ecuaciones de factor de fricción explícito para tuberías. Educación Química, 25(2), 128-134. Recuperado de https://doi.org/10.1016/S0187$893 \times(14) 70535-X$

Anderson, A. G. (1947). Fluid flow diversion: A summary and bibliography of literature. In: St. Anthony Falls Hydraulic Laboratory (1). Minneapolis, USA: University of Minnesota.

Aronov, I. Z. (1950). Teploobmen i gidravlicheskoe soprotivlenie $v$ izognutykh trubakh (Heat Exchange and Hydraulic Resistance in Bent Pipes) (Doctorate's Thesis) Kiev, Ukraine: Kiev Polytechnic Institute.

Arun, G., Kumaresh, S. P., Natarajan, S., \& Kulasekharan, N. (2020). Study of flow behaviour in sharp and mitred pipe bends. Materials Today: Proceedings, 27(3), 2101-2108. Recuperado de https://doi.org/10.1016/j.matpr.2019.09.076

Balch, L. R. (1913). Investigation of hydraulic curve resistance, experiments with three-inch pipe. Bulletin of the University of Wisconsin, 403(7), 253.

Bariviera, D., Frizzone, J. A., \& Rettore, O. (2014). Dimensional analysis approach to estimate local head losses in microirrigation connectors. Irrigation Science, 32, 169-179. Recuperado de https://doi.org/10.1007/s00271-013-0424-y

Beij, K. H. (1938). Pressure losses for fluid flow in $90^{\circ}$ pipe bends. Journal 
Tecnología y

Ciencias $₫$ Agua
2021, Instituto Mexicano de Tecnología del Agua

Open Access bajo la licencia CC BY-NC-SA 4.0

(https://creativecommons.org/licenses/by-nc-sa/4.0/)

of Research of the National Bureau of Standards, 21(1), 1-18. Recuperado de

https://doi.org/http://dx.doi.org/10.6028/jres.021.001

Blevins, R. D. (1984). Applied fluid dynamics handbook. New York, USA: Van Nostrand Reinhold.

Brater, E. F., King, H. W., Lindell, J. E., \& Wei, C. Y. (1996). Handbook of Hydraulics (7th ed.). New York, USA: McGraw-Hill Professional.

Brightmore, A. W. (1907). Loss of pressure in water flowing through straight and curved pipes. Minutes of the Proceedings of the Institution of Civil Engineers, 169(1907), 315-336. Recuperado de https://doi.org/10.1680/imotp.1907.17203

CFE, Comisión Federal de Electricidad. (1983). Manual de diseño de obras civiles, Hidrotecnia: Hidráulica, A.2.3 Conducciones a presión. México, DF, México: Comisión Federal de Electricidad, Instituto de Investigaciones Eléctricas.

Chen-Tzu, Y. (1969). Loss of pressure in water flowing through $90^{\circ}$ pipe bend (Kansas State University). Recuperado de https://archive.org/stream/lossofpressurein00yuch/lossofpressurein o0yuch_djvu.txt

Chowdhury, R. R., Biswas, S., Alam, M. M., \& Islam, A. K. M. S. (2016). Turbulent flow analysis on bend and downstream of the bend for different curvature ratio. AIP Conference Proceedings, 1754, 1-6. Recuperado de https://doi.org/10.1063/1.4958380

Crane Co. (1982). Flow of fluids throuh valves, fittings and pipe. In: Technical Paper No. 410. New York, USA: Crane Company. 
Tecnología y

Ciencias $₫$ Agua
2021, Instituto Mexicano de Tecnología del Agua

Open Access bajo la licencia CC BY-NC-SA 4.0

(https://creativecommons.org/licenses/by-nc-sa/4.0/)

Daneshfaraz, R., Rezazadehjoudi, A., \& Abraham, J. (2018). Numerical investigation on the effect of sudden contraction on flow behavior in a 90-degree bend. KSCE Journal of Civil Engineering, 22(2), 603-612. Recuperado de https://doi.org/10.1007/s12205-017-1313-3

Dang, Z., Yang, Z., Yang, X., \& Ishii, M. (2018). Experimental study of vertical and horizontal two-phase pipe flow through double 90 degree elbows. International Journal of Heat and Mass Transfer, 120, 861869.

Recuperado

de

https://doi.org/10.1016/j.ijheatmasstransfer.2017.11.089

Daugherty, R. L., \& Ingersoll, A. C. (1954). Fluid Mechanics, with Engineering Applications (6th ed.). Daugherty, R. L. \& Ingersoll, A. C. (eds.). New York, USA: McGraw-Hil.

Davis, G. J. (1911). Investigation of hydraulic curve resistance. Bulletin of the University of Wisconsin, 403(6), 115.

Du, X., Wei, A., Fang, Y., Yang, Z., Wei, D., Lin, C., \& Jin, Z. (2020). The effect of bend angle on pressure drop and flow behavior in a corrugated duct. Acta Mechanica. Recuperado de https://doi.org/10.1007/s00707-020-02716-5

Dutta, P., \& Nandi, N. (2015). Study on pressure drop characteristics of single phase turbulent flow in pipe bend for high Reynolds Number. ARPN Journal of Engineering and Applied Sciences, 10(5), 2221-2226. Recuperado de http://www.arpnjournals.com/jeas/research_papers/rp_2015/jeas_0 315_1746.pdf

Elbatran, A. H., Yaakob, O. B., Ahmed, Y. M., \& Shabara, H. M. (2015). 
Ciencias $₫$ Agua
2021, Instituto Mexicano de Tecnología del Agua

Open Access bajo la licencia CC BY-NC-SA 4.0

(https://creativecommons.org/licenses/by-nc-sa/4.0/)

Operation, performance and economic analysis of low head microhydropower turbines for rural and remote areas: A review. Renewable and Sustainable Energy Reviews, 43, 40-50. Recuperado de https://doi.org/10.1016/j.rser.2014.11.045

Evdomikov, I. F. (1940). Opyty po otsasyvaniyu pogranichnogo sloya v aerodinamicheskikh trubakh bol'shikh skorostei (Experiments on the suction of the boundary layer in high-velocity wind tunnels). Trudy TSAGI, 506, n.p.

Franzini, J. B., \& Finnemore, E. J. (1999). Mecánica de fluidos con aplicaciones en ingeniería (9th ed.). Madrid, España: McGraw-Hill / Interamericana de España.

Friman, M., \& Levy, A. (2019). Bends pressure drop in hydraulic conveying. Advanced Powder Technology, 30(8), 1484-1493. Recuperado de https://doi.org/10.1016/j.apt.2019.04.025

Fuentes, O. A., \& Rosales, I. L. (2004). Estimación de pérdidas locales de energía en tomas domiciliarias de agua potable. Ingeniería Hidráulica en México, 19(1), 65-73. Recuperado de file:///C:/Users/Jose Juan/Downloads/1009-1109-1-PB.pdf

Gibson, A. H. (1930). Hydraulics and its applications (4th ed.). New York, USA: D. Van Nostrand Company.

Gontsov, N. G., Marinova, O. A., \& Tananaev, A. V. (1984). Turbulent flow around a bend in a circular pipe. Hydrotechnical Construction, 18(12), 596-602. Recuperado de https://doi.org/10.1007/BF01440641

Gülich, J. F. (2014). Fluid dynamic principles. In: Centrifugal pumps (3rd ed.) (pp. 1-41). Recuperado de https://doi.org/10.1007/978-3-642- 
Tecnología y

Ciencias Agua
2021, Instituto Mexicano de Tecnología del Agua

Open Access bajo la licencia CC BY-NC-SA 4.0

(https://creativecommons.org/licenses/by-nc-sa/4.0/)

40114-5_1

Haase, D. (1953). Allgemeine Wärmetechnik. Nos(11-12), n.d.

Hager, W. H. (2010). Waste water hydraulics, theory and practice. In:

Springer Science and Business Media (2nd. ed.). Recuperado de https://doi.org/10.1007/978-3-642-11383-3

Herning, F. (1966). Stoffströme in Rohrleitungen (4t ed.). Düsseldorf, Germany: VDI.

Herwig, H., \& Wenterodt, T. (2011). Second law analysis of momentum and heat transfer in unit operations. International Journal of Heat and Mass Transfer, 54, 1323-1330. Recuperado de https://doi.org/10.1016/j.ijheatmasstransfer.2010.11.055

Hofmann, A. (1929). Der Verlust in $90^{\circ}$ Rohrkrümmern mit gleiclibleibendem Kreisquerschnitt. Mitteilungen Des Hydraulischen Instituts Der Technischen Hochschule München, 3, 45-67.

Hsu, B. E. (1981). Flow of fine particle suspensions in bends, fittings and valves (Texas Tech University). Recuperado de https://ttuir.tdl.org/ttuir/bitstream/handle/2346/19704/31295002382256. pdf?sequence $=1$ \&isAllowed $=\mathrm{y}$

Idel'chik, I. E. (1966). Handbook of hydraulic resistance, coefficients of local resistance and of friction (No. 6630). Recuperado de https://doi.org/AEC-tr- 6630

Idel'chik, I. E. (1953). K voprosu o vliyanii chisla Re i sherokhovatosti na soprotivienie izognutykh kanalov (The Influence of the Reynolds 
Tecnología y

Ciencias $₫$ Agua
2021, Instituto Mexicano de Tecnología del Agua

Open Access bajo la licencia CC BY-NC-SA 4.0

(https://creativecommons.org/licenses/by-nc-sa/4.0/)

Number and the roughness on the resistance of bent channels). Promyshlennaya Aerodinamika, 4, 177-194.

Ito, H. (1960). Pressure losses in smooth pipe bends. Journal of Basic Engineering, 82(1), 131-140. Recuperado de https://doi.org/10.1115/1.3662501

Ito, H. (1959). Friction factors for turbulent flow in curved pipes. Transactions of the American Society of Mechanical Engineers, Series D, Journal of Basic Engineering, 81, 123-134.

Ito, H. (1956). On the pressure losses for turbulent flow in smooth pipe bends. In: Reports of The Institute of High Speed Mechanics, 6, 55102.

Jia, W., \& Yan, J. (2020). Pressure drop characteristics and minimum pressure drop velocity for pneumatic conveying of polyacrylamide in a horizontal pipe with bends at both ends. Powder Technology, 372, 192-203.

Recuperado

de

https://doi.org/10.1016/j.powtec.2020.06.004

Kast, W. (2010). L1 Pressure drop in single phase flow. In: e. V. VDI (Ed.), VDI Heat Atlas (2nd ed.) (pp. 1053-1116). Recuperado de https://doi.org/10.1007/978-3-540-77877-6_70

Kilkovsky, B., Jegla, Z., \& Stehlik, P. (2011). Comparison of different methods for pressure drop calculation in $90^{\circ}$ and $180^{\circ}$ Elbows. Chemical Engineering Transactions, 25(1), 243-248. Recuperado de https://doi.org/10.3303/CET1125041

King, H. W., Wisler, C. O., \& Woodburn, J. G. (1980). Hidráulica. México, DF, México: Trillas. 
2021, Instituto Mexicano de Tecnología del Agua

Open Access bajo la licencia CC BY-NC-SA 4.0

(https://creativecommons.org/licenses/by-nc-sa/4.0/)

Kirchbach, H. (1929). Der Energieverlust in Kniestücken (The energy loss in elbows, in German). Mitteilungen Des Hydraulischen Instituts Der Technischen Hochschule, 3, 43-64.

Liu, S. H., Xue, J., \& Fan, M. (2013). The calculation of mechanical energy loss for incompressible steady pipe flow of homogeneous fluid. Journal of Hydrodynamics, 25(6), 912-918. Recuperado de https://doi.org/10.1016/S1001-6058(13)60440-0

Lourenco, A., \& Xin, G. (2017). Design and development of an experimental apparatus for the study of minor loss of the mini-scale fittings (University of Macau). Recuperado de http://oaps.umac.mo/handle/10692.1/152

Maria-Di, F. (2000). Design and off design pipe network geothermal power plant analysis with power pipe simulator. Energy Conversion and Management, 41, 1223-1235. Recuperado de https://doi.org/10.1016/S0196-8904(99)00177-6

Mataix, C. (2010). Mecánica de fluidos y máquinas hidráulicas (2nd ed.). México, DF, México: Alfaomega Editorial.

Mays, L. W. (2001). Water Resources Engineering. New York, USA: John Wiley \& Sons, Inc.

Menon, E. S., \& Menon, P. S. (2010). Pressure loss through piping systems. In: Menon, E. S. \& Menon, P. S. (eds.). Working Guide to Pumps and Pumping Stations (pp. 69-111). Recuperado de https://doi.org/https://doi.org/10.1016/B978-1-85617-828$0.00004-4$

Miller, D. S. (1990). Internal Flow Systems (2nd ed.). Cranfield-Bedford, 
Tecnología y

Ciencias Agua
2021, Instituto Mexicano de Tecnología del Agua

Open Access bajo la licencia CC BY-NC-SA 4.0

(https://creativecommons.org/licenses/by-nc-sa/4.0/)

UK: BHRA.

Munson, B. R., Okiishi, T. H., Huebsch, W. W., \& Rothmayer, A. P. (2013). Fundamentals of fluid mechanics. In: Fowley, D., Welter, J., \& Holm, K. (eds.). Biofluid Mechanics (7th ed.). Recuperado de https://doi.org/10.1016/B978-0-12-381383-1.00002-3

Nasir, B. A. (2014). Design considerations of micro-hydro-electric power plant. Energy Procedia, 50, 19-29. Recuperado de https://doi.org/10.1016/j.egypro.2014.06.003

Nekrasov, B. B. (1954). Gidravlika (Hydraulics). Russia: VVA.

Pashkov, N. N., \& Dolqachev, F. M. (1985). Hidráulica y máquinas hidráulicas. Moscú, Rusia: Mir Moscú.

Perumal, K., \& Ganesan, R. (2016). CFD modeling for the estimation of pressure loss coefficients of pipe fittings: An undergraduate project. Computer Applications in Engineering Education, 24(2), 180-185. Recuperado de https://doi.org/10.1002/cae.21695

Pigott, R. J. S. (1957). Losses in Pipe and Fittings. Transactions of the American Society of Mechanical Engineers, 79, 1767-1783.

Pigott, R. J. S. (1950). Pressure losses in tubing, pipe, and fittings. Transactions of the American Society of Mechanical Engineers, 72, 679-688.

Reghunathan, R., Son, S. W., Suryan, A., \& Kim, H. D. (2019). Study on reduction in pressure losses in pipe bends using guide vanes. Journal of Visualization, 22(4), 795-807. Recuperado de https://doi.org/10.1007/s12650-019-00561-w 
Tecnología y

Ciencias $₫$ Agua
2021, Instituto Mexicano de Tecnología del Agua

Open Access bajo la licencia CC BY-NC-SA 4.0

(https://creativecommons.org/licenses/by-nc-sa/4.0/)

Richter, G. (1936). Gidravlika truboprovodov (Hydraulics of Pipelines). Moscow, Russia: ONTI.

Richter, H. (1930). Der Druckabfall in gekrümmten glatten Rohrleitungen. Forschungsarbeiten Auf Dem Gebiete Des Ingenieurwesens, VDI , 338, $30-47$.

Saldarriaga, J. G. (2016). Hidráulica de tuberías: abastecimiento de agua, redes $y$ riegos (3a. ed.). Recuperado de http://books.google.com/books?id=-76BPgAACAAJ\&pgis $=1$

SARH, Secretaría de Agricultura y Recursos Hidráulicos. (1984). Obras de toma para presas de almacenamiento. México, DF, México: Dirección General de Obras Hidráulicas y de Ingeniería Agrícola para el Desarrollo Rural, Secretaría de Agricultura y Recursos Hidráulicos.

Schmandt, B., Iyer, V., \& Herwig, H. (2014). Determination of head change coefficients for dividing and combining junctions: A method based on the second law of thermodynamics. Chemical Engineering Science, 111, 191-202. Recuperado de https://doi.org/10.1016/j.ces.2014.02.035

Schoder, E. W. (1908). Loss coefficient experiments in pipe bends. Transactions of the American Society of Civil Engineers, 34, 416-445.

Schubart, W. (1929). Der Verlust in Kniestücken bei glatter und rauher Wandung (The loss in elbows with smooth and rough wall, in German). Mitteilungen Des Hydraulischen Inst. Der Technischen Hochschule, 3, 81-99.

Sesma, J., Molina-Martínez, J. M., Cavas-Martínez, F., \& FernándezPacheco, D. G. (2015). A mobile application to calculate optimum drip 
Tecnología y

Ciencias $₫$ Agua
2021, Instituto Mexicano de Tecnología del Agua

Open Access bajo la licencia CC BY-NC-SA 4.0

(https://creativecommons.org/licenses/by-nc-sa/4.0/)

irrigation laterals. Agricultural Water Management, 151, 13-18. Recuperado de https://doi.org/10.1016/j.agwat.2014.09.026

Shames, I. H. (1995). Mecánica de fluidos (3a ed.). Bogotá, Colombia: McGraw-Hill Book Interamericana.

Simon, A. L. (1986). Hidráulica práctica. México, DF, México: Limusa.

Sotelo, G. A. (2013). Hidráulica general. Recuperado de https://doi.org/10.1017/CBO9781107415324.004

Tripathi, N. M., Portnikov, D., Levy, A., \& Kalman, H. (2019). Bend pressure drop in horizontal and vertical dilute phase pneumatic conveying systems. Chemical Engineering Science, 209, 115228. Recuperado de https://doi.org/10.1016/j.ces.2019.115228

Trueba, C. S. (1986). Hidráulica (23a ed.). México, DF, México: Continental S. A.

Turian, R. M., Hsu, F. L., \& Selim, M. S. (1983). Friction losses for flow of slurries in pipeline bends, fittings, and valves. Particulate Science and Technology, 1(4), 365-392. Recuperado de https://doi.org/10.1080/02726358308906383

USACE, United States Army Corps of Engineer. (1980). Engineering and design: Hydraulic design of reservoir outlet works. Recuperado de http://www.publications.usace.army.mil/Portals/76/Publications/Eng ineerManuals/EM_1110-2-1602.pdf

USBR, United States Bureau of Reclamation. (1985). Diseño de presas pequeñas (13a ed.). México, DF, México: Continental S. A.

Villegas-León, J. J., López-Lambraño, A. A., Morales-Nava, J. G., Pliego- 
Díaz, M., Fuentes, C., \& López-Ramos, A. (2016). Equations to determine energy losses in sudden and gradual change of direction. Recent Advances in Fluid Dynamics with Environmental Applications, 4, 465-475. Recuperado de https://doi.org/10.1007/978-3-31927965-7

Vogel, H. D. (1933). Head losses in various types of pipe bends. Reston, USA: The U.S. Army Corps of Engineers Waterways Experiment Station.

Wasielewski, R. (1932). Verluste in glatten Rohrkrümmern mit kreisrundem Querschnitt bei weniger als $90^{\circ}$ Ablenkung. Mitteilungen Des Hydraulischen Instituts Der Technischen Hochschule München, 5, 53-57.

Weisbach, J. (1855). Experimentelle Hydraulik. Freiberg, Germany: n.e.

White, C. M. (1929). Streamline flow through curved pipes. Proceedings of the Royal Society of London.Series A, Containing Papers of a Mathematical and Physical Character, 123(792), 645-663. Recuperado de https://doi.org/10.1098/rspa.1929.0089

White, F. M. (2008). Mecánica de fluidos (6a ed.). Madrid, España: McGraw-Hill.

Yildirim, G., \& Singh, V. P. (2010). A MathCAD procedure for commercial pipeline hydraulic design considering local energy losses. Advances in Engineering Software, 41, 489-496. Recuperado de https://doi.org/10.1016/j.advengsoft.2009.10.007

Yoo, D. H., \& Singh, V. P. (2010). Explicit design of commercial pipes with secondary losses. Journal of Hydro-Environment Research, 4(1), 37- 
45. Recuperado de https://doi.org/10.1016/j.jher.2009.12.003

Yuce, M., \& Muratoglu, A. (2015). Hydrokinetic energy conversion systems: A technology status review. Renewable and Sustainable Energy Reviews, 43, 72-82. Recuperado de https://doi.org/10.1016/j.rser.2010.06.016

Zmrhal, V., \& Schwarzer, J. (2009). Numerical simulation of local loss coefficients of ventilation duct fittings. Eleventh International IBPSA Conference, July 27-30, 2009, 1761-1766. Recuperado de http://www.ibpsa.org/proceedings/BS2009/BS09_1761_1766.pdf 